\title{
DEVELOPMENT OF SPATIAL FUNCTION IN RATS AND ASSOCIATED C-FOS PATTERNS IN THE HIPPOCAMPAL CA1 AND CA3 REGIONS
}

$$
\text { by }
$$

\author{
Rachel Louise Comba
}

\begin{abstract}
A thesis submitted to the Faculty of Graduate Studies and Postdoctoral Affairs in partial fulfillment of the requirements of the degree of
\end{abstract}

Masters of Science

in

Neuroscience

\author{
Carleton University \\ Ottawa, Ontario
}

(C) 2013

Rachel Louise Comba 


\begin{abstract}
The hippocampus shows connectivity-based changes during development. The current work investigated the contribution of performance and cognitive factors in the emergence of spatial function during development. Experiment one showed a sensitive developmental period exists between 16 days old (p16) and p24. Experiment two found impaired performance on spatial learning following NMDAr blockade. Experiment three explored one-day of water maze training at p16,p18, or p20 and spatial learning was established only at p20. Quantification of c-Fos positive cells in CA3 and CA1 revealed a main effect of age, CA3 also revealed a main effect of training. Lastly, rats were trained on the dry maze at: p16, p17, p18, p19, or p20. Groups p19 and p20 demonstrated spatial learning. Quantification of c-Fos positive cells in CA1 revealed a main effect of age. This suggests that spatial memory mechanisms emerge at p19/p20 independently from performance factors and are based on hippocampal function.
\end{abstract}




\section{Acknowledgements}

I would like to express my gratitude to my supervisor Dr. Matthew Holahan for his guidance and expertise throughout my degree. I appreciate the opportunity I was given coming from an Arts degree and the belief Dr. Holahan had in me to succeed in this program.

To Catherine Smith and Brianne Wartman, for mentorship and guidance in the lab. Your patience and assistance allowed me to learn techniques, become independent and able to mentor new students in the lab. I truly appreciate everything you both did for me.

To Dr. David Mumby at Concordia University and his student Nicole Gervais, thank you for continuing this collaboration and inviting me to do some work at Concordia University. It was a great experience to work in a different lab and share techniques.

To the Department of Neuroscience, faculty, staff and fellow students, thank you all for the classes, mentoring, encouragement and friendships.

Lastly, thank you to my friends and family. I have received so much support over the course of this degree. I would like to dedicate this work to my mother who has been the most thoughtful, selfless, caring mom I could ever ask for. Thank you. 


\section{Table of Contents}

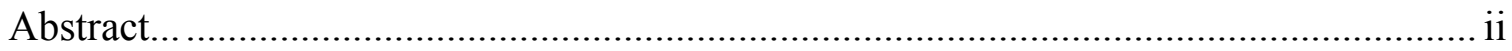

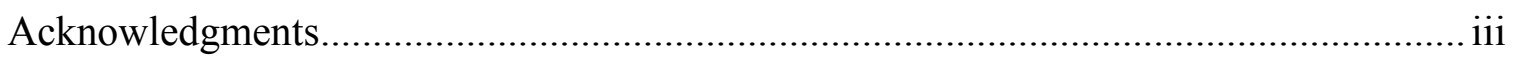

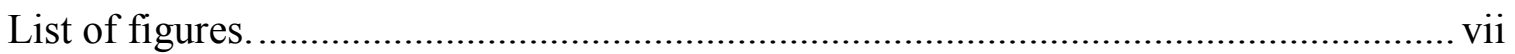

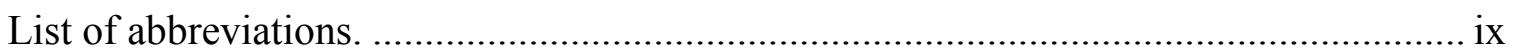

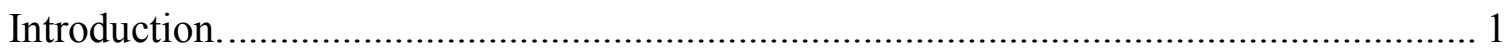

Overview of development. ......................................................................... 1

The hippocampus. .................................................................................... 1

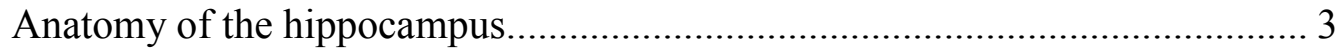

LTP and the hippocampus........................................................................ 5

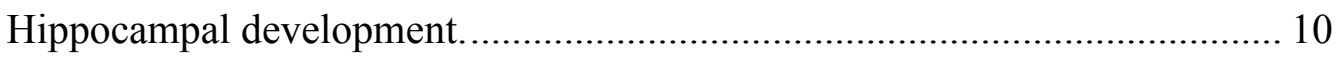

Altering hippocampal development. ........................................................... 12

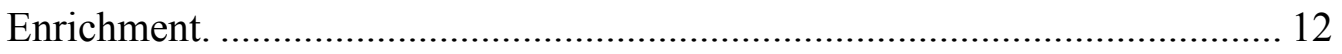

Effects of behavioural training during development...................................... 14

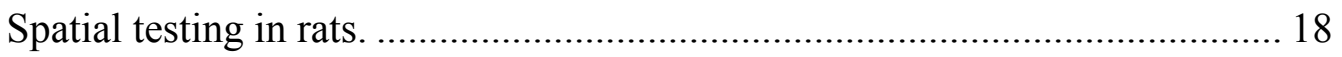

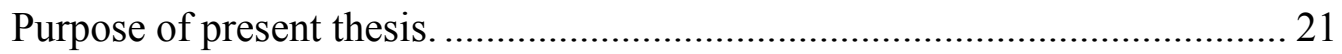

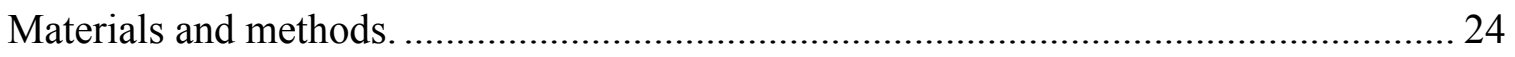

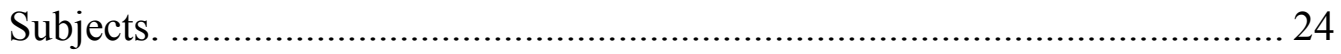

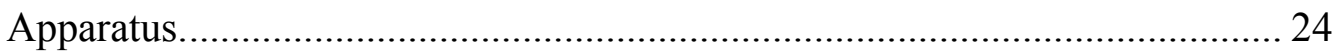

Water maze.....................................................24

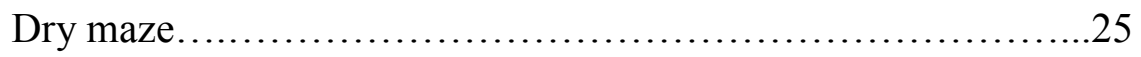

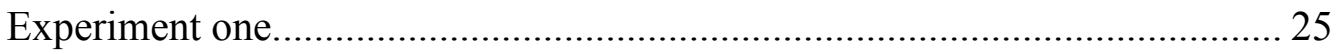

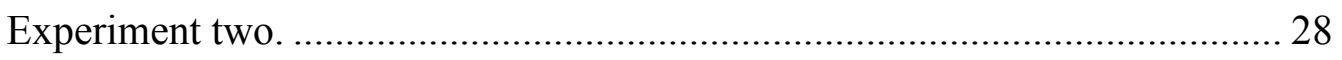

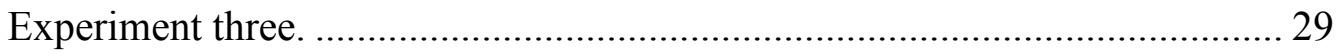




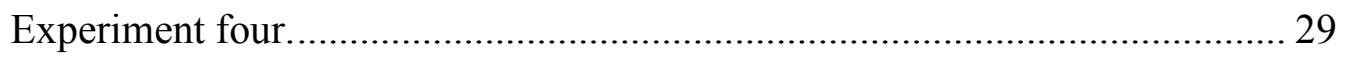

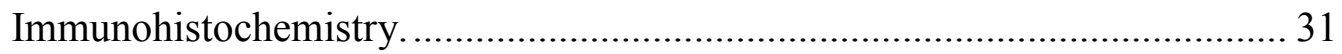

Histology .................................................... 31

c-Fos............................................................

c-Fos Quantification...........................................33

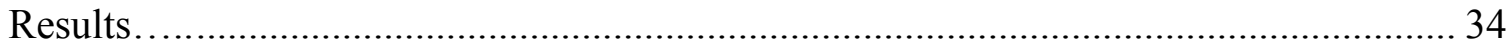

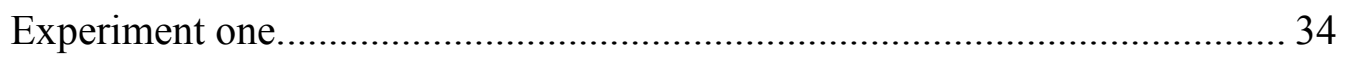

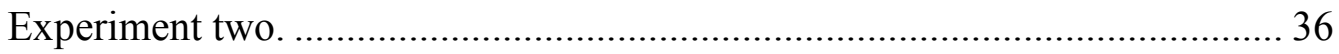

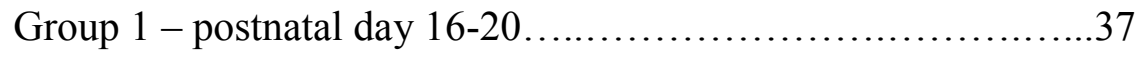

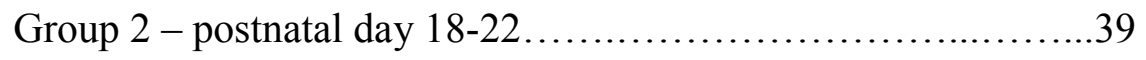

Group 3 - postnatal day $20-24 \ldots \ldots \ldots \ldots \ldots \ldots \ldots \ldots \ldots \ldots \ldots . . . \ldots 1$

All groups...................................................... 43

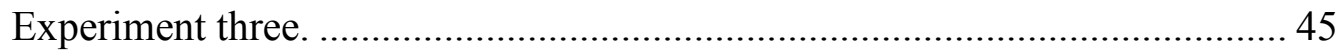

Behavioural Results..........................................45

Immunohistochemistry......................................48

Immunohistochemical results $(\mathrm{CA} 3) \ldots \ldots \ldots \ldots \ldots \ldots \ldots \ldots \ldots \ldots . \ldots \ldots$

Immunohistochemical results $(\mathrm{CA} 1) \ldots \ldots \ldots \ldots \ldots \ldots \ldots \ldots \ldots . . . \ldots 52$

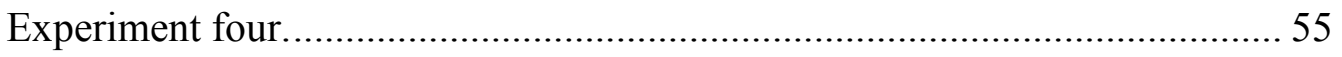

Behavioural results........................................... 55

Immunohistochemical results (CA3) ..........................57

Immunohistochemical results $(\mathrm{CA} 1) \ldots \ldots \ldots \ldots \ldots \ldots \ldots \ldots \ldots \ldots . . .61$

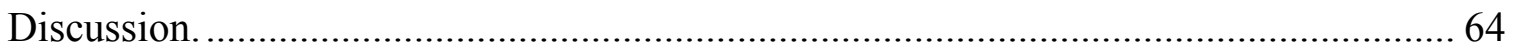

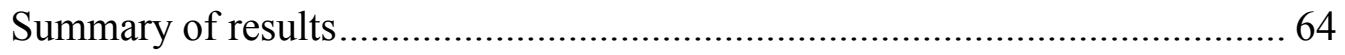

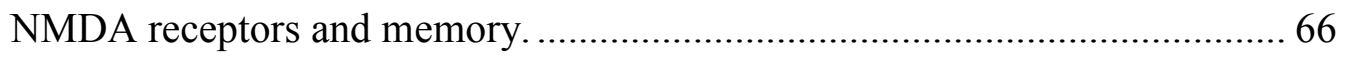


Emergence of spatial functions.

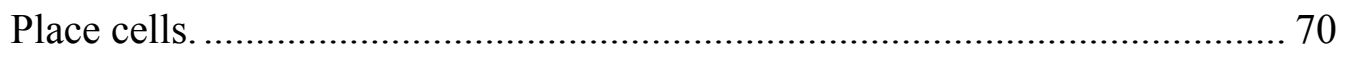

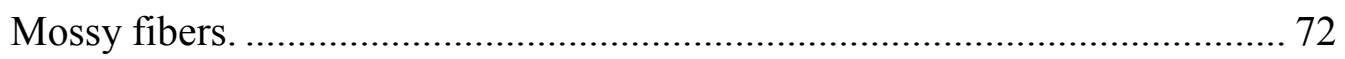

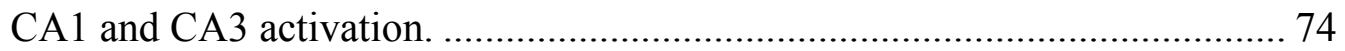

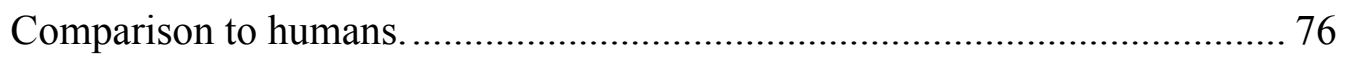

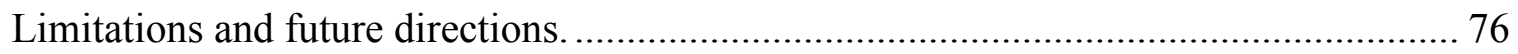

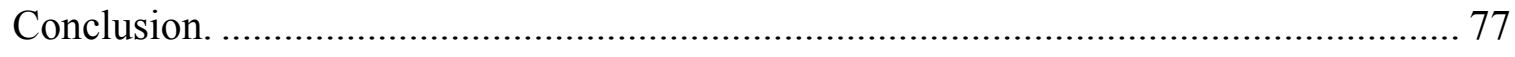

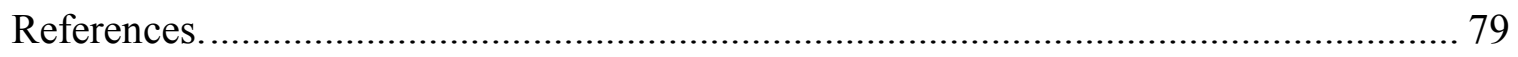




\section{List of Figures}

Figure 1. Modified water maze task. .................................................................... 27

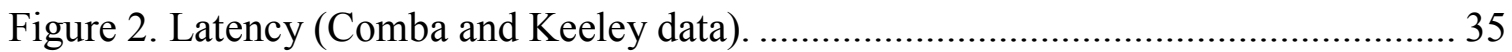

Figure 3. Latency and speed data for rats treated with MK-801 or saline from p16-p20..38

Figure 4. Latency and speed data for rats treated with MK-801 or saline from p18-p22..40

Figure 5. Latency and speed data for rats treated with MK-801 or saline from p20-p24..42

Figure 6. Latency to reach the platform averaged across eight trials for each groups start

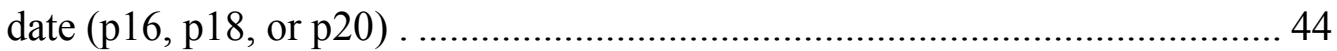

Figure 7. Latency to reach the hidden platform for one day only at start dates: p16, p18 and $\mathrm{p} 20$. 46

Figure 8. Speed to reach the hidden platform for one day only at start dates: p16, p18 and $\mathrm{p} 20$.

Figure 9. Quantification of c-Fos positive cells in the CA3 region of the hippocampus with and without water maze training for $\mathrm{p} 16, \mathrm{p} 18$ and $\mathrm{p} 20$

Figure 10. Representative images for c-Fos positive cells in the CA3 and CA1 50

Figure 11. Traced area of the CA3 (water maze)....................................................... 51

Figure 12. Quantification of c-Fos positive cells in the CA1 region of the hippocampus with and without water maze training for $\mathrm{p} 16, \mathrm{p} 18$ and $\mathrm{p} 20$ 53

Figure 13. Traced area of the CA1 (water maze) ..................................................... 54

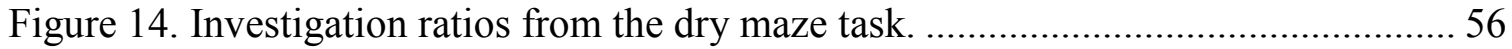

Figure 15. Quantification of c-Fos staining in the CA3 region of the hippocampus following dry maze training and testing at: p16, p17, p18, p19 or p20. ........ 58

Figure 16. Representative images for c-Fos positive cells in the CA3 and CA1 ............ 59

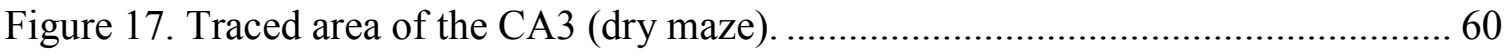


Figure 18. Quantification of c-Fos staining in the CA1 region of the hippocampus following dry maze training and testing at: p16, p17, p18, p19 or p20 ........... 62

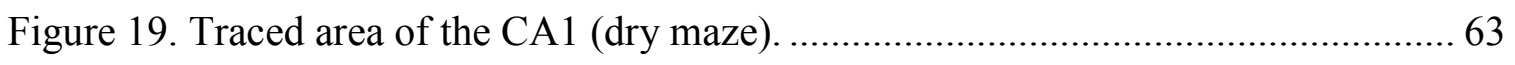




\section{List of Abbreviations}

a-amino-3-hydroxy-5-methyl-4-isoxazolepropionate (AMPA)

2R,4R,5S-2-amino-4,5-(1,2-cyclohexyl)-7-phosphonoheptano acid (NPC17742)

3-(2-carboxypiperazin-4-yl) propyl-1-phosphonic acid (CPP)

3, 3'-diaminobenzidine (DAB)

Analysis of variance (ANOVA)

Animal free blocker (AFB)

Canadian Council on Animal Care (CCAC)

Cornu ammonis/Ammon's horn (CA)

D-aminophosphonovalerate (D-APV or D-AP5)

Dentate gyrus (DG)

Distilled water $\left(\mathrm{dH}_{2} \mathrm{O}\right)$

Dizocilpine (MK-801)

Dry maze (DM)

Embryonic day (E)

Ethanol (EtOH)

Hydrogen peroxide $\left(\mathrm{H}_{2} \mathrm{O}_{2}\right)$

Intraperitoneal (IP)

Investigation ratio (IR)

Long-Evans rat (LER)

Long-term potentiation (LTP)

N-methyl-D-aspartate (NMDA)

N-methyl-D-aspartate receptor (NMDAr)

No water maze (NOWM)

Novel object-in-place (NOIP)

Phosphate buffer (PB)

Phosphate buffered saline (PBS) 
Phosphate buffered saline with Triton X (T-PBS)

Phosphorylated extracellular regulated kinase 1 (pERK1)

Postnatal day (p)

Stratum lucidum (SL)

Stratum oriens (SO)

Tetraethylammonium (TEA)

Water maze (WM) 


\section{Introduction}

\section{Overview of development}

Non-genetic experiences in the womb and shortly after birth can radically alter the normal developmental progression of the brain and the behaviours it produces. If the brain is adversely affected by toxins, development may be compromised, producing detrimental effects throughout the lifespan of the organism. Neural development can also be facilitated as when some form of enrichment occurs during critical or sensitive periods. As the brain develops, neurons become more efficiently connected through synaptogenesis and pruning. With this increased connectivity, increasingly complex mental health functions emerge. The developing nervous system requires an extensive amount of pruning and remodelling in order to achieve its final, fully functioning adult configuration (Kantor \& Kolodkin, 2003). These connectivity-based changes occur in spurts or sensitive periods that can be observed brain-wide or in localized brain regions (Fenoglio et al., 2006) and are determined by a combination of innate genetic factors and responses to external stimuli (Sur \& Rubenstein, 2005). Insults, as well as enrichment, that occur during these sensitive periods can often have a long-lasting, irreversible beneficial or detrimental effects.

\section{The hippocampus}

One localized brain region that shows widespread connectivity-based changes during sensitive developmental periods is the hippocampus. The hippocampus is a structure that lies in the medial temporal lobe of the human brain and has long been studied for its prominent role in learning and memory (Scoville \& Milner, 1957; Morris et al., 1982; Nadel \& Bohbot, 2001). Patient H.M. is one of the most famous cases in the 
world of neuroscience with respect to memory research. This patient initially presented as an individual who suffered from severe epilepsy. When no other methods were successful to reduce the burden of his epilepsy, Dr. William Scoville came to the conclusion that surgery was the only option. In 1953, Dr. Scoville performed a bilateral medial temporal lobe resection in an attempt to cure H.M.'s severe and debilitating epilepsy (Scoville \& Milner, 1957). The resection was said to extend eight centimetres back from the tips of the temporal lobes (Scoville \& Milner, 1957; Corkin, 1984). Many brain structures were removed: the prepyriform gyrus, the amygdala, the uncus, the hippocampus, and parahippocampal gyrus (Corkin, 1984). As a result of this surgery, H.M. suffered from anterograde amnesia (inability to form new long-term memories) and partial retrograde amnesia (the inability to remember things before the time of the insult; in H.M.'s case, the surgery). H.M. suffered only partial retrograde amnesia as he forgot about two years of his life before the time of the surgery. However, his anterograde amnesia was very severe as he could no longer form new long-term memories. Once his attention was diverted from what he was trying to remember, he would forget the information completely and immediately (Scoville \& Milner, 1957). This behavioural change was the only severe symptom shown after H.M.'s surgery as his IQ remained largely the same. This led many neuroscientists to believe that these brain structures were crucial for certain memory functions.

H.M.'s memory impairment was selective to his declarative memory. This means that he could not remember someone's name after meeting them the previous day, where he currently resided, or whether he had brushed his teeth that morning (Corkin, 1984). Interestingly, H.M. could perform just as well as control subjects on motor skill learning, 
cognitive skill learning, and perceptual skill learning showing he was capable of implicitly remembering things (Milner et al., 1998). This meant that although he could not consciously remember, he could remember things unconsciously. One task he showed this memory ability for was a mirror drawing task. H.M needed to receive instructions detailing how to complete the task each time he was asked to perform, but oddly, his performance improved each time. The interest in memory formation and hippocampal function began to flourish after this bizarre and groundbreaking case leading researchers to further investigate the role of the medial temporal lobes in declarative memory formation and retrieval.

\section{Anatomy of the Hippocampus}

The hippocampal formation is made up of four units: the dentate gyrus, the hippocampus proper (CA1, CA2 \& CA3), the subicular complex, and the entorhinal cortex (Amaral \& Witter, 1989). The hippocampus proper is of particular interest to this paper and is divided into three sections: the CA1, CA2, and CA3 (CA comes from cornu ammonis, or Ammon's horn). The CA2 and CA3 contain large pyramidal cell bodies. The dentate gyrus has a V or U shape and remains similar in structure throughout different levels of the hippocampus. The dentate gyrus receives projections from the entorhinal cortex and this pathway is known as the perforant path (Teyler \& DiScenna, 1984). This pathway is unidirectional as the dentate gyrus does not project back to the entorhinal cortex. The main cell type of the dentate gyrus is granule cells which give rise to axons called mossy fibers. These granule cells exist in a single layer, around five to ten cells thick (Teyler \& DiScenna, 1984). The main transmitter used by these granule cells is glutamate. The mossy fibers connect with the pyramidal cells of the CA3 (Teyler 
\& DiScenna, 1984). The pyramidal cells in the CA3 are the main source of input to the CA1 via the Schaffer collateral axons (Teyler \& DiScenna, 1984). Continuing in this fashion, the CA1 projects to the subiculum which does not project back to the CA1. There are two areas of the CA3 of particular interest, the stratum lucidum (SL) and the stratum oriens (SO). The SL is formed by efferent fibers, i.e. the mossy fibers, that project from the dentate gyrus (Blackstad, 1956; Raisman et al., 1965). The SO is composed of the basal dendrites of the pyramidal cells and is the location of the commencement of Schaffer collaterals that project to the CA1 (Raisman et al., 1965).

As mentioned, the main transmitter used by granule cells in the dentate gyrus is glutamate. Glutamate is the major excitatory neurotransmitter in the central nervous system and is involved in many brain functions, including synaptic plasticity (Cotman et al., 1988; Wisden \& Seeburg, 1993) cell survival and death, long-term potentiation and depression, as well as development of neuronal and glial cells (Nakanishi \& Masu, 1994). Glutamate acts on two different receptor types: ionotropic and metabotropic (Nakanishi \& Masu, 1994; Salt \& Eaton, 1996). Ionotropic receptors are ion channels that have a binding site for the neurotransmitter and a pore that opens when the neurotransmitter is bound. These receptors allow for quick, and in the case of glutamate, excitatory synaptic transmission (Chen \& Wyllie, 2006). There are three separate subgroups of ionotropic receptors based on their selective agonists: N-methyl-D-aspartate (NMDA), kainate, and a-amino-3-hydroxy-5-methyl-4-isoxazolepropionate (AMPA). Both the kainate and AMPA receptors can be activated with either kainate and/or AMPA, but not by NMDA (Salt \& Eaton, 1996). The NMDA receptor is unique because it requires the binding of both glutamate and the co-agonist glycine for the channel to open (Chen \& Wyllie, 2006). 
AMPA receptors are primarily permeable to sodium, whereas the NMDA receptors have higher calcium permeability (Malenka, 1994; Salt \& Eaton, 1996). AMPA receptors allow the influx of sodium into the cell almost immediately and are faster acting than NMDA receptors, as they do not have the magnesium block (Malenka, 1994).

Anatomically, NMDAr are distributed throughout the brain. The highest levels are found in the Schaffer collateral pathway of the hippocampus, which, as mentioned previously project from the pyramidal cells of the CA3 to the CA1, as well as the lateral septum, where the hippocampal fibers terminate (Cotman et al., 1988). It is believed that these receptors are crucial in learning and memory as they have such a high concentration in the hippocampus.

\section{LTP and the hippocampus}

In 1949, Donald Hebb described a process which is now referred to as Hebbian plasticity. The term is used to describe the idea that simultaneous activation of two cells increases their effectiveness. This strengthening between cells was thought to be crucial for learning and memory. Bliss and Lomo (1973) performed a study where they examined the effects of short periods of stimulation on the dentate area in anaesthetized rabbits. They used two electrodes, one to stimulate the perforant path and the other to record in the dentate area. They showed that excitatory postsynaptic potentials could be potentiated for a long period of time after only a few conditioning trials. These trials consisted of single or short periods of stimulation within one second. The amount of stimulations varied from one to 15 per second. They found an increase in amplitude of excitatory postsynaptic potentiation when rabbits received 10 to 15 stimulations per second for several seconds, and this increase remained in some cases for six to eight 
hours after the last conditioning trial. The increase in amplitude began to drop gradually but remained above the pre-conditioning value 10 hours after the last trial. The association and strengthening between cells was now shown experimentally through electrical stimulation of the perforant path in the hippocampus. They found that when they stimulated the perforant path with weak electrical currents, there were similar weak responses in the granule cells of the dentate. However, when they applied high frequency stimulation to the perforant path, they found that afterwards, when the weak electrical input was applied, there was now a strong response in the granule cells of the dentate (Bliss \& Lomo, 1973). This was the first evidence that the hippocampus could change its responses to weak inputs after a high frequency input and thus, laid the groundwork for the investigation into the neural mechanisms underlying this potentiated electrical response. Because activity in the hippocampus was strengthened for long periods of time from repeated stimulation, it could provide a model for information storage.

Long-term potentiation (LTP) is the idea that high-frequency stimulation increases synaptic plasticity and efficiency in the ability of two neurons to communicate with each other. The induction of LTP is mediated through the activation of the NMDAr complex (Collingridge et al., 1983; Bliss \& Collingridge, 1993; Moser \& Moser, 2000; Ge et al., 2010). For LTP to occur, depolarization of the cell must be strong enough to displace the magnesium ion. A single excitatory post-synaptic potential is not strong enough for the magnesium ion to be displaced. The magnesium ion is displaced when the inside of the cell becomes sufficiently depolarized. The neurotransmitter is released and it binds to both NMDA and non-NMDA (i.e. kainate or AMPA) receptors. The non- 
NMDA receptors respond and sodium enters the cell causing it to depolarize. Once enough sodium has entered the cell to bring the electrical charge to a level of $-30 \mathrm{mV}$, the magnesium block is removed and calcium ions flow into the cell through the channel of the NMDA receptor (Collingridge \& Bliss, 1987).

Hippocampal LTP is commonly known to be induced through the calcium influx through opening of NMDA channels (Hanse \& Gustafsson, 1994). Following on this logic, blocking NMDA receptors through pharmacological antagonists would not only block the influx of calcium but also LTP. Evidence for this comes from a study carried out by Malenka (1991) where he observed the likelihood of short- or long-term potentiation in the presence of a competitive NMDAr antagonist Daminophosphonovalerate (D-APV or D-AP5). In the presence of a high dose $(25 \mu \mathrm{M})$ of D-AP5, the potentiation following a single tetanus of 0.25 seconds to one second in duration never lasted more than 60 seconds. This length of potentiation is not enough to be classified as either short- or long-term potentiation. Using a lower dose of D-AP5 $(2 \mu \mathrm{M})$ led to short-term potentiation following the same single tetanus with the same length. However, long-term potentiation was not achieved in the presence of any amount of D-AP5. Therefore, without the influx of calcium into the cell, LTP cannot occur. Another study showed that blocking NMDA receptors in rat hippocampal slices prevented long-lasting synaptic growth that occurred normally in dendritic regions of CA1 neurons localized near the stimulating electrode (Maletic-Savatic et al., 1999). These results suggest that LTP stability relies heavily on NMDA receptor activation and the associated calcium influx. 
There are two types of long-term potentiation: NMDA receptor-dependent and NMDA receptor-independent (Aniksztejn \& Ben-Ari, 1991; Grover \& Teyler, 1995). In the CA1 area of the hippocampus, LTP is induced by an influx of calcium into the pyramidal neurons via NMDA receptors (Malenka, 1991; Bliss and Collingridge, 1993; Maletic-Savatic et al., 1999). In both NMDA receptor-dependent and -independent LTP, an initial increase in calcium concentration occurs in postsynaptic neurons (Aniksztejn \& Ben-Ari, 1991). Therefore, although induction of these forms of LTP occur through different receptors, they lead to the same result of increased calcium, and presumably the same subsequent steps leading to expression and maintenance of potentiation. Aniksztejn and Ben-Ari (1991) studied the different forms of LTP using various antagonists. They found that tetraethylammonium (TEA), which blocks the potassium current leaving the

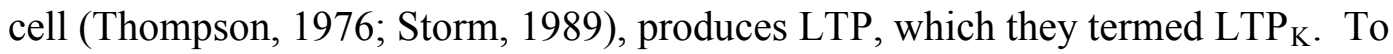
examine whether LTP is NMDAr-dependent or -independent, they used NMDAr antagonist D-AP5. They found that the antagonist blocked electrical LTP (evoked stimulation of the Schaffer collaterals and recorded in the stratum radiatum of the CA1) but it did not block this new form of LTP, $\mathrm{LTP}_{\mathrm{K}}$. In this manner, LTP was induced through calcium influx through voltage-dependent calcium channels, instead of NMDA receptors (NMDAr; see also Grover and Teyler (1995). Therefore, both NMDArdependent and -independent LTP exist and they share the influx of calcium despite their different use of mechanisms.

Long-term potentiation is a model used to explain the synaptic basis of learning and long-term memory storage. LTP is expressed both pre- and post-synaptically (Bliss \& Collingridge, 1993; Malenka, 1994). A form of pre-synaptic potentiation comes from 
experiments which showed increased release of neurotransmitters from the hippocampus before and after LTP induction (Bliss et al., 1986). It could be that the number of synaptic vesicles in the presynaptic terminals increase, or the size of the vesicles increase (Bailey \& Kandel, 1993). There also exists evidence for post-synaptic expression (Lynch et al., 1976; Kauer et al., 1988). Lynch and colleagues (1976) found that tetanic stimulation on the Schaffer collaterals increases the receptiveness of the postsynaptic neuron. Therefore, the expression of LTP is likely a combination of both pre- and postsynaptic changes.

LTP is thus an extremely important part in the memory process and therefore, blocking LTP from occurring could be detrimental in memory storage. Research shows that NMDA receptor antagonist dizocilpine or (+)-5-methyl-10,11-dihydro-5Hdibenzo[a,d]cyclohepten-5,10-imine maleate (MK-801) not only blocks hippocampal LTP, but also impairs spatial learning and memory (Ahlander et al., 1999). MK-801 sits in the receptor channel and therefore prevents calcium influx. Researchers (Morris et al., 1986) blocked NMDA receptors with antagonist D-AP5 to examine the effects on LTP as well as behavioural changes. The NMDAr antagonist blocked LTP as well as memory formation. The rats that were chronically injected with NMDAr antagonists showed marked impairment in spatial learning and memory when participating in spatial tasks. However, there are cases where NMDAr are blocked by antagonists but the rat still shows spatial learning and memory (Bannerman et al., 1995; Saucier and Cain, 1995; Moser \& Moser, 2000). Saucier and Cain (1995) performed a study where rats were given a potent and specific NMDAr antagonist, 2R,4R,5S-2-amino-4,5-(1,2-cyclohexyl)-7phosphonoheptano acid (NPC17742). The NPC17742 blocked dentate gyrus LTP 
although, they found that if rats received pretraining then they showed no impairment when performing the water maze task. The pretrained experimental group which received the NMDAr antagonist performed just as well as pretrained controls in finding the location of the hidden platform in terms of latencies and swim paths. Therefore, the deficit seen in rats given NMDAr antagonists can be reversed if the rat receives pretraining. This suggests that the hippocampus is needed for spatial learning but NMDA receptors are not needed in all circumstances, as in the case with pretraining.

\section{Hippocampal development}

The hippocampal formation undergoes extremely rapid growth rates during embryonic stages and early postnatal life. Bayer (1980) studied the morphogenesis of the hippocampal region of the rat during embryonic and early postnatal life and found that it becomes recognizable at embryonic day 16 (E16). Embryonic days 16 and 17 are when the most rapid growth rates occur as the hippocampal formation increases in volume by 1900\% (Bayer, 1980). This growth slows down but continues into early postnatal (p) life and maintains its development by approximately $12 \%$ daily between p 7 and p 21 (Bayer, 1980; Holahan et al., 2007).

One hippocampal subregion that shows a high degree of axonal structural malleability during development and adulthood is the CA3 region (Holahan et al., 2007). Within the CA3, there are mossy fiber projections from the granule cells in the dentate gyrus that mainly make synaptic contacts on the apical dendrites (located in the SL) of the CA3 pyramidal neurons and to a lesser extent, the mossy fibers make contacts on the basal dendrites (located in the SO) of CA3 pyramidal neurons (Raisman et al., 1965; Teyler \& DiScenna, 1983). 
Previous research has examined the natural expansion and retraction of mossy fibers during development (Holahan et al., 2007). Using Timm's stain, a marker for zinc (Danscher, 1981) which is in high concentrations at mossy fiber terminals (Frederickson et al., 1983) to analyze the mossy fiber projection, Holahan and colleagues (2007) noted staining in the SL of the CA3 region on p12 in the Long-Evans rat. On p18, staining in the $\mathrm{SO}$ of the $\mathrm{CA} 3$ region appeared and staining in both the SL and SO remained throughout development and well into adulthood in the Long-Evans rat. The outgrowth of the mossy fibers occurred mostly during the p18-p24 developmental period suggesting morphological changes in the brain, notably the hippocampus, may be aiding the rat in various spatial tasks.

When spatial memories are formed in adult rats, there appears to be significantly more mossy fiber terminal projections that innervate the SO (Ramirez-Amaya et al., 1999; Holahan et al., 2006). Researchers investigated whether synaptogenesis occurred in adult rats in response to mossy fiber LTP induction in vivo (Escobar et al., 1997) as it did following brief seizure episodes (Ben-Ari \& Represa, 1990). Escobar and colleagues (1997) found that LTP occurred in all adult rats, including those treated with a competitive NMDAr antagonist, suggesting that mossy fiber LTP can be induced independent from NMDAr function. In addition to this finding, there was also an increase in Timm's staining in the SO of the rats that received high-frequency mossy fiber stimulation. Moreover, high-frequency mossy fiber stimulation sufficient to produce mossy fiber LTP led to an increase in the number of granule cells in the dentate gyrus (Derrick et al., 2000). Therefore, in the adult, the hippocampus undergoes synaptogenesis and neurogenesis after electrical stimulation and behavioural training. 


\section{Altering Hippocampal Development}

The hippocampus has been noted as a brain region that undergoes extensive morphological changes such as synaptogenesis and neurogenesis during development and into adulthood (Escobar et al., 1997; Derrick et al., 2000; Holahan et al., 2007).

One way of initiating morphological changes in the hippocampus in the developing rat include enrichment paradigms. Enrichment can be in the form of behavioural training (Keeley et al., 2010; Wartman et al., 2012) or housing conditions (Fiala et al., 1978; Kempermann et al., 1997) both of which induce morphological changes in the hippocampus similar to those seen in adults.

\section{Enrichment}

Previous research has shown similarities in behaviour between rats with hippocampal damage (Kimble, 1963) and isolation-reared rats (Fiala et al., 1978). This suggests that housing conditions may be important factors in altering the developmental aspects of the hippocampus. Fiala and colleagues (1978) examined hippocampal morphogenesis in developing and adult rats following exposure to complex or isolated environments for four weeks after weaning (developing rats) and for twelve weeks beginning at p145 (adult rats). Hippocampal morphogenesis was studied using Golgi techniques to count and measure dendritic field length and width. The complex environment consisted of multiple rats housed together in a large mesh cage with plastic, wood, and metal toys. The isolated environment consisted of single-housed rats in stainless-steel cages so they could not see or interact with other rats. They found that rats exposed to a complex environment - independent from age - showed a significantly 
greater number of lower order dendritic branches (i.e. those close to the cell body) in granule cells than those exposed to an isolated environment.

Exposure to an enriched environment, such as a cage with tunnels, a wheel, toys, etc. (Kempermann et al., 1997), leads to morphological changes in the hippocampus. These changes are comprised of increases in the number of dendritic spines (Fiala et al., 1978) and an increase in the volume of the granule cell layer (Kempermann et al., 1997). It is well known that neurons undergo changes during development at the level of the synapse (Markus et al., 1987) and dendrites (Petit et al., 1988). However, Petit and colleagues (1988) noted that repetitive stimulation in the hippocampus leads to an increase in the synaptic density of neurons in adult rats. This put forward the idea that there is a potential for plasticity in the hippocampus continuing into adulthood.

Kempermann and colleagues (1997) found a significant improvement in latencies to find the platform on the Morris water maze task for mice that were exposed to an enriched environment. The mice in this study also showed an increase in the number of granule cells in the dentate gyrus. These granule cells promote the formation and projection of mossy fibers into the $\mathrm{SO}$ of the $\mathrm{CA} 3$ region in the hippocampus. These increased intrahippocampal pathways are NMDAr independent. The newly formed NMDAr independent intrahippocampal pathways explain why rats treated with NMDAr antagonists and also receive pretraining have intact spatial learning and memory abilities. Therefore, animals exposed to enriched environments perform better on spatial learning tasks because of these structural changes within the hippocampus. 


\section{Effects of behavioural training during development}

There is a key developmental period that has been examined in Long-Evans rats (Rudy et al., 1987; Holahan et al., 2007; Keeley et al., 2010). Rudy and colleagues found rats had the ability to use distal spatial cues to locate a hidden platform in the water maze task at p20. However, they found the p17 rats could only perform the task using proximal cues, i.e. a visible platform. This suggests the skills necessary to learn and navigate a spatial location using distal cues are not fully developed until p20. Following this, Keeley and colleagues (2010) trained rats on a modified water maze task from p16 to $\mathrm{p} 26$ to investigate the sensitive developmental period Rudy and colleagues (1987) noted in their research. They found there was some learning that occurred between p16 and p19, but there was a large drop in average latencies on the hidden platform water maze task from $\mathrm{p} 19$ to $\mathrm{p} 20$. This improvement suggests that either the rats were forming spatial memories of their environment or there was something else occurring in the body and/or the brain that was assisting the rat on this spatial learning task. One possibility is the brain may still be developing and there could be more mossy fiber innervation (Ramirez-Amaya et al., 1999; Holahan et al., 2006) or, it is possible that the body itself is becoming stronger with age and there is more muscle mass which is aiding on completing this both cognitive and physical task.

Immunohistochemical results from Keeley and colleagues (2010) found decreased phosphorylated extracellular regulated kinase 1 (pERK1) staining in the CA3 for rats that received pretraining on the water maze. Phosphorylated ERK1 is a marker for cells that have undergone some growth process, therefore the decrease in staining suggests that ERK1 may have been phosphorylated during pretraining and persisted into adulthood. 
Thus, in this fashion, the nonpretrained rats had higher levels of staining because their first exposure to the task was during adolescence, therefore beginning the phosphorylation process at a later age. More immunohistochemical results revealed increased synaptophsyin staining in the CA3 SO in the male pretrained groups. As mentioned previously, CA3 SO staining suggests there are structural changes occurring in the hippocampus and they are associated with improved spatial learning and memory on behavioural tasks, such as the water maze.

Similar work has demonstrated comparable improvements on the water maze task in preadolescent rats between p19 and p22 (Wartman et al., 2012). This study aimed at differentiating whether the improvement on the water maze task was due to task familiarity or spatial familiarity. Task familiarity features include swimming ability and habituation to the water, where as spatial familiarity is referring to memory function where the rat is able to learn and remember distal cues and locate the hidden platform. To examine whether the differences were due to task or spatial familiarity, two tasks were used: the water maze and the dry maze. In order to compare the results from the different tasks, both tasks used the same pool and the spatial cues remained constant.

The six different conditions were juvenile pretraining on the water maze (WM) then testing during adolescence on either the water maze (WM/WM) or the dry maze (WM/DM); pretraining on the dry maze (DM) then testing during adolescence on either the water maze $(\mathrm{DM} / \mathrm{WM})$ or dry maze $(\mathrm{DM} / \mathrm{DM})$; or no pretraining $(\mathrm{NT})$ and then testing during adolescence on either the water maze (NT/WM) or dry maze (NT/DM). These groups were created to examine differences between task and spatial familiarity. For example, improved performance during adolescence in the WM/WM only would 
suggest that the task is due to task familiarity because the rat has habituated to the water, and has improved their swimming ability. Improved performance in the DM/WM group during adolescence would suggest that spatial familiarity is predominant because the rat has not experienced the performance aspects of the water maze (i.e. swimming) but they have learned and remembered the spatial cues around the room. The results indicated that rats exposed to either the dry maze or the water maze during pretraining showed improved performance on the water maze. This suggests spatial familiarity contributes to enhanced learning on the water maze task. Consistent with these findings, the immunohistochemical results also showed an increase in synaptophysin staining in groups that received exposure to spatial cues during preadolescence. Therefore, these changes in connectivity may be essential for learning based on spatial familiarity, but not as important for task familiarity learning.

The water maze task is physically demanding as the rat must be able to swim for 60 seconds at maximum and climb onto the platform. Although the pool for the preadolescent rats is much smaller than that for the adults, it remains a difficult task. It is also a very cognitively demanding task as the rat needs to use external spatial cues to locate the hidden platform. However, there is sufficient evidence to indicate that the rats are more than capable to perform this task during pre-adolescence, i.e. p16 to p20 (Bolles \& Woods, 1964; Gard et al., 1967; Altman \& Sudarshan, 1975). Researchers have noted that once eyelids begin to open, which naturally occurs at about p14 to p16, there is an increase in certain behaviours such as exploration, sniffing, climbing and rearing, among many others (Bolles \& Woods, 1964). Exploratory behaviours like those seen in a dry maze task require both motor and cognitive maturation (Foreman \& Altaha, 1992). 
Therefore, for the dry maze to be completed during the rats juvenile period, physical and cognitive abilities are necessary.

A study by Altman and Sudarshan (1975) examined the development of locomotor skills in the rat. They found that by $\mathrm{p} 12$, rats can raise one of their forelimbs without losing balance and by $\mathrm{p} 15$, rats can lift their entire bodies and engage in exploratory behaviours (such as rearing) in the dry maze. Rearing is when the rat is raised on its hindlegs and it represents a form of investigatory response in the open field, or dry maze. They also noted that by $\mathrm{p} 16$, the rats are able to run quickly with few hindlimb slips. They seem to be in control of their body and therefore, should be capable of performing more complex motor tasks such as swimming in the water maze.

Another important observation made by these researchers (Altman \& Sudarshan, 1975) and others (Gard et al., 1967) is that certain behaviours can be elicited earlier than they would naturally occur, i.e. rats can perform certain tasks if there are aversive stimuli. For example, in the Altman and Sudarshan study (1975), the aversive stimulus was the rats being isolated from their siblings. They performed an experiment where the rats needed to traverse complicated paths (i.e. narrow bridges) to return to their home cage. The rats performed well starting around p10 and could perform the task perfectly, without falling, from p16 onwards. As mentioned above, rats develop the ability to run with few hindlimb slips at p16 when observing their natural behaviour, but they are able to perform a more complex task (traversing narrow bridges) at the same age when facing the aversive stimulus of being isolated from their siblings.

In accordance with this, Gard and colleagues (1967) performed similar work with aversive stimuli. They exposed pre-adolescent rats to two different conditions to 
determine when certain motor behaviours develop and if they can be elicited earlier in the presence of aversive stimuli. Each animal was exposed to both conditions separated by a period of six hours. These conditions were counterbalanced to ensure one was not affecting the other. A meshed-net covered frame was placed on top of a can that contained either $100 \mathrm{~mL}$ of cold water or concentrated ammonia (which evaporated for five minutes to allow for alleviation of toxins). The rat was placed on top of the can and their behavioural patterns were recorded during their two separate one minute trials. In the water condition, rats began "pivoting" (includes movement of head, trunk, and all legs) on $\mathrm{p} 8$ whereas the rats in the ammonia condition pivoted at $\mathrm{p} 4$. They found this earlier occurrence for other complex behaviours in rats during their ammonia exposure, compared to their water condition. The rats may therefore have the necessary neural circuits needed to perform these types of behaviours before they are outwardly observed at a later age. Therefore at p16 and onwards, the rats should be both physically and cognitively (Gard, et al., 1967; Altman \& Sudarshan, 1975) able to participate in tasks such as walking and exploring in the dry maze and swimming in the water maze.

\section{Spatial testing in rats}

The Morris water maze is a key behavioural procedure used to investigate spatial learning and memory in rodents (Morris et al., 1982). The water maze is a large circular pool that contains a hidden platform for the rat to escape. It is situated in a room with spatial cues around the wall which permits the rat to learn and remember the location of the platform in relation to these distal cues. The platform remains in the same quadrant for a series of trials to allow the rat to learn the location of the platform. To ensure that the rat is actively remembering where the platform is located, it is placed just below the 
water level, guaranteeing the platform is hidden. The rat is placed in the pool from different start locations and allowed to swim freely for 60 seconds or until it reaches the platform (whichever is first). The time it takes for the rat to reach the platform is recorded and shorter latencies imply that the rat is learning and remembering the location of the platform.

An alternative water maze is also occasionally used in which the platform location changes (Keeley et al., 2010). On the first day of testing the platform remains in a fixed location for all trials. On all other days of testing, the location of the platform is the same as the previous day for the first trial and then moves to a new location for trials two through eight. This ensures that learning is continuing throughout the training period and that the task remains novel and difficult. This forces the rats to learn and remember the spatial cues to a greater extent than when the standard water maze task is used.

Another behavioural task that measures spatial learning and memory without the swimming performance component is the dry maze. This task measures the recognition of spatial relations between two objects. It consists of two phases: the familiarization phase and the test phase (Gaskin et al., 2010). During the familiarization phase, two identical objects are placed in two adjacent quadrants and the rat is brought in the maze and allowed to explore for seven minutes. This phase occurs three times before the test phase. There are different retention delays between familiarization and testing and this can lead to differences when studying the effects of brain damage or pharmacological treatments (Gaskin et al., 2003; Gaskin et al., 2010). During the test phase, one of the objects is moved to a new quadrant. The rat is placed in the maze for five minutes during this phase and is again, allowed to explore. This task is based on rats' tendency to 
investigate a familiar object in a novel location more than a familiar object in the same location hence tapping into the ability to detect spatial rearrangements. It is a measure of spatial learning and memory as the objects are identical but one has moved locations and therefore, when the rat detects the change in the spatial arrangement of the two objects, it will increase the time spent exploring the object in the new location (Mumby et al., 2002; Gaskin et al., 2010). There are different ways to analyze the performance of the rats on this task, and in the case of this paper, the investigation ratio (IR) is used (Clark et al., 2000; Gaskin et al., 2003). The investigation ratio is calculated by dividing the time spent investigating the moved object relative to total object investigation. A larger investigation ratio means that the rat spent more time investigating the moved object than the not moved object (Clark et al., 2000; Gaskin et al., 2003)

Previous research shows that lesions or temporary inactivation of the hippocampus leads to poor performance on spatial memory tasks like the water maze (Morris et al., 1982). Rats with hippocampal lesions perform significantly worse on the water maze task as they show significantly longer latencies to locate the platform and their swim paths are very different from controls. However, these deficits may be overcome when the animals are provided with pretraining (Morris, 1989; Saucier \& Cain, 1995). The ability for the pretrained rats to surmount these deficits may be due to performance, as these rats have received more practice in the maze and have become more comfortable with the task (i.e. swimming) and the new room (i.e. stress). Another possibility is that the pretraining may be leading to increased communication between neurons and therefore stronger connections within the hippocampal networks. These structural changes in the hippocampus provide support for its involvement in memory 
formation. Previous research showed that pretraining protocols lead to increases in CA3 SO synaptophysin staining (Keeley et al., 2010) and Timm's staining (Holahan et al., 2006) in the hippocampus, indicative of memory formation. Therefore, it can be inferred that mossy fibers are sensitive to different spatial tasks and structurally change with more training and are therefore an ideal system to examine while carrying out spatial learning and memory tasks during developmental periods.

\section{Purpose of Present Thesis}

To understand more about learning and memory, the current study first examined this key sensitive developmental period by replicating the findings from Keeley and colleagues (2010) and Wartman and colleagues (2012). Past research examining this period has shown a big drop in latencies on the water maze between p18 and p20 (Keeley et al., 2010; Wartman et al., 2012). Therefore, the first experiment in the current study aimed to replicate these previous findings by training Long-Evans rats on the modified water maze during this sensitive developmental period. It was hypothesized that rats would improve in performance over trials and days and that a similar drop in latencies would occur between p18 and p20 to the Keeley and colleagues (2010) and Wartman and colleagues (2012) data.

The second experiment aimed at deconstructing this sensitive period into three subgroups to allow more critical examination of what was occurring during this time frame. These subgroups were: $\mathrm{p} 16$ to $\mathrm{p} 20 ; \mathrm{p} 18$ to $\mathrm{p} 22$; and $\mathrm{p} 20$ to $\mathrm{p} 24$. This experiment also investigated the effects of NMDA receptor blockade on performance in a spatial task for all three subgroups to examine whether spatial learning is NMDA receptor dependent or independent during development. The non-competitive NMDA receptor antagonist, 
MK-801, was used for this experiment. It was hypothesized that saline rats would perform better on the task than MK-801 treated rats and the older and more trained rats would perform best on the task.

A third experiment was used to investigate the functional changes in the brain and behavioural changes that occurred at developmental milestones. Long-Evans rats either received one day of training or no training on the water maze on the same start days of our last experiment, i.e. p16, p18, or p20. It was hypothesized that rats who received one day of training would show more functional changes in the brain when compared to their non-trained counterparts. It was also hypothesized that the older rats would perform better on the water maze than their younger counterparts.

The fourth and final experiment aimed at examining whether the drop in latencies on the water maze that were observed in previous studies carried out by Keeley and colleagues (2010) and Wartman and colleagues (2012) during this developmental period was due to changes in the brain, or changes in the body. This portion of the study was accomplished by testing the rats on a dry maze task. This task was used as it is not as physically demanding as the water maze task. The rationale behind this task was the rats would perform similarly to the water maze task in that we would see a larger investigation ratio (IR) in the older rats. As mentioned previously, a larger IR suggests the rat spent more time investigating the moved object when compared to the object that did not move from the familiarization phase to the test phase. The prediction was that the trend would be similar to the drop in latencies seen in the water maze. Long-Evans rats were trained and tested on the dry maze on one of five days (p16, p17, p18, p19, or p20) to attempt to replicate the water maze findings on dry land in a task that does not require 
such physical skills like swimming, to complete. It was hypothesized that larger investigation ratios would be observed around p19, consistent with previous research using the water maze task (Keeley et al., 2010; Wartman et al., 2012). Furthermore, it was hypothesized that older rats would show more functional changes in the brain compared to their younger counterparts.

Experiments 3 and 4 also had an immunohistochemical portion to examine if cFos, an immediate early gene protein product, is activated following spatial learning on both the water maze and dry maze tasks. Research by Morgan and Curran (1986) has demonstrated using an agonist for the voltage-dependent calcium channel that c-Fos induction occurs following the activation of these channels and the resultant influx of calcium. Further research shows increased levels of c-Fos positive cells following seizures (Dragunow \& Robertson, 1987), kindling (Herrera \& Robertson, 1996), and some forms of learning (Tischmeyer et al., 1990). Furthermore, both the CA1 and CA3 will be analyzed to determine whether these regions are differentially active depending on the task, as past research using odour discrimination tasks (Hess et al., 1995) as well as associative learning tasks (Gilbert \& Kesner, 2003) show multiple modes of functioning in the hippocampus. 


\section{Materials and methods}

\section{Subjects}

One hundred and fourteen male Long-Evans rats (LERs) were used over all four experiments. Seventy-nine LERs were used in the first three experiments and were born in the animal-care facility at Carleton University. The remaining thirty-five LERs were used in the fourth experiment and were born in the animal-care facility at Concordia University. The day the pups were born was marked as p0. Pups were weaned on their first day of training. For all experiments, the animals were group-housed. All animals at Carleton University were housed in a temperature controlled vivarium in polycarbonite cages with a 12 hour light-dark cycle: lights on $8 \mathrm{~h} 00$, lights off $20 \mathrm{~h} 00$. The animals at Concordia University were also housed in a temperature controlled vivarium in polycarbonite cages with a 12 hour light-dark cycle, lights off at $8 \mathrm{~h} 00$ and lights on at 20h00. Food and water were provided ad libitum. All experiments were conducted in accordance with the Canadian Council on Animal Care (CCAC) guidelines and approved by the Carleton University Animal Care Committee or the Concordia University Animal Care and Use Committee.

\section{Apparatus}

Water maze (Carleton University)

The pool was opaque, white polypropylene with a submerged platform $2 \mathrm{~cm}$ below the water surface. Water temperature averaged $23 \pm 1{ }^{\circ} \mathrm{C}$ throughout behavioural testing. The water maze was a white, circular, polypropylene pool measuring $124 \mathrm{~cm}$ in diameter, $31 \mathrm{~cm}$ in height and filled with water to a depth of $25 \mathrm{~cm}$. The platform was made of clear Plexiglas and measured $11 \mathrm{~cm}$ in diameter. Distal visual cues were present 
during all trials. Rat movements in the pool were tracked using the HVS Image 2100 Tracking System (HVS Image, Buckingham, UK). The water was quickly cleaned after each trial and the pool was drained and refilled every other day.

Dry Maze (Concordia University)

The dry maze was $122 \mathrm{~cm}$ in diameter and the Lexan walls were $10.5 \mathrm{~cm}$ high, 2 mm thick. The floor of the maze was opaque white and the walls were transparent. The two stimulus objects used for the dry maze were turquoise water bottles made of glazed ceramic and were approximately $20 \mathrm{~cm}$ high and the widest circumference approximately $23 \mathrm{~cm}$. A small glass jar ( $7 \mathrm{~cm}$ high, $20 \mathrm{~cm}$ in circumference) was secured to the bottom of each object with epoxy. The jar lids were inverted and fastened to the plastic floor to allow the objects to be secured in place by screwing the jars into the lids. Holes were drilled at the center of each quadrant to accommodate the lids and to allow the counterbalancing of the object's positions. When not in use, the holes were covered with a small piece of circular white tape. After each trial, the objects and maze were wiped down with water, spreading any smell over the entire maze and objects. At the beginning of each day, the floor of the maze and objects were washed with a $70 \%$ alcohol solution and the Lexan walls were cleaned with standard window cleaner.

\section{Experiment 1: Juvenile water maze learning and comparison to previous findings}

One group of seven rats were trained on the modified water maze from p16 to p24. Previous research by Keeley and colleagues (2010), as well as Wartman and colleagues (2012) showed a significant decrease in latencies between p16 and p26 to reach the platform in a water maze task. This first experiment was designed to replicate the findings that a sensitive developmental period exists. The seven animals were trained 
on the modified water maze for nine days (p16-p24). All animals were given eight trials a day. Each trial began from a different start point on the pool's perimeter and the same sequence of start locations was used each day. On the first day, the platform remained in the same quadrant for all eight trials. For the remaining days, for Trial one, the platform remained in the same position as the previous days' Trials two through eight. On the second through eighth trial, the platform was moved to a different quadrant (see Figure 1 for illustration). Latency and speed to reach the platform were recorded for all trials. If an animal did not reach the platform within 60 seconds, they were guided to it. Animals remained on the platform for 30 seconds before being removed, dried, and placed into a holding cage for an additional 30 seconds. Following the final trial on each day, every animal was dried with a towel and placed in another holding cage on a heating pad in the housing room for 15 to 20 minutes before being returned to their home cage. Following their last day of training, the rats were placed into a carbon dioxide chamber for several seconds until they were no longer fully aware and then euthanized by decapitation. 


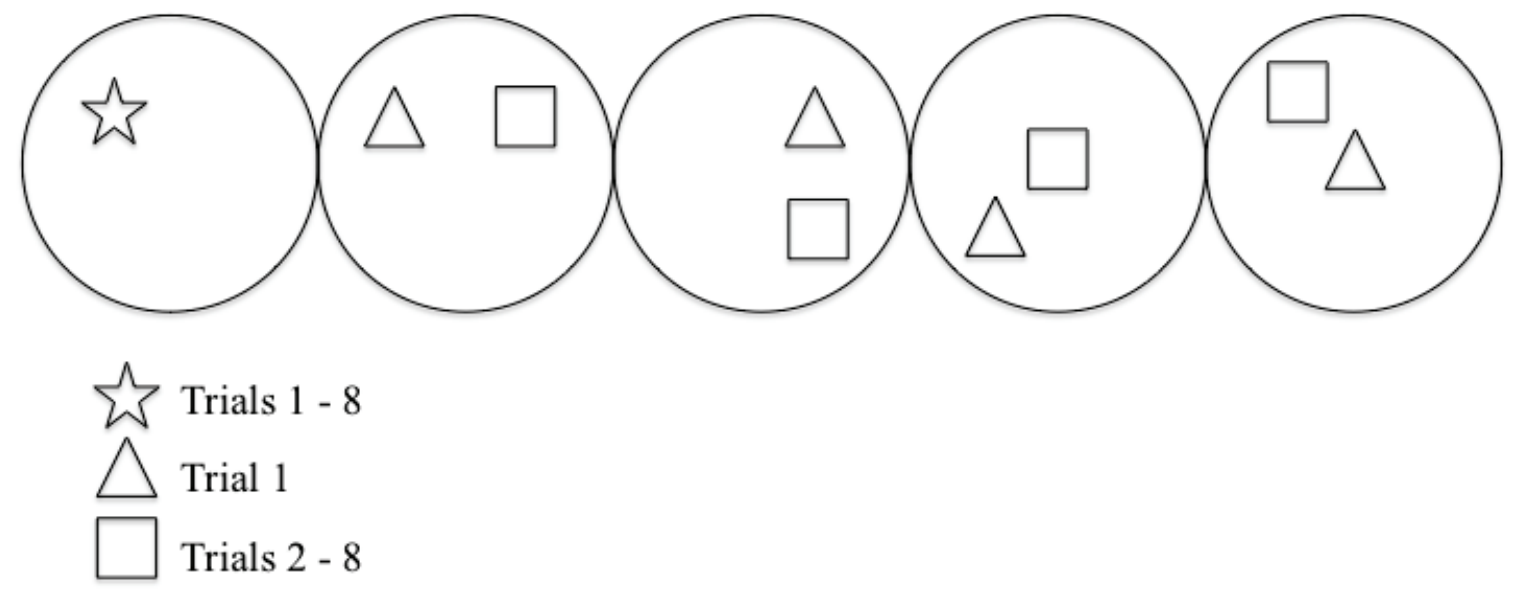

Figure 1. Platform locations across 5 days of testing. On the first day, the platform remained in the same location across all trials. On the first trial of the other days of testing, the platform remained in the same location as the previous day's eighth trial. On the second through eighth trials, the platform was moved to a new location in the pool. 


\section{Experiment 2: Effect of NMDAr blockade on juvenile spatial learning}

This experiment was carried out for two main purposes. The first was to determine whether the learning curve observed in research by Keeley and colleagues (2010) and Wartman and colleagues (2012) was due to practice or the emergence of spatial function. Therefore, the sensitive developmental period observed in these previous studies was broken down into three subgroups starting at $\mathrm{p} 16, \mathrm{p} 18$, or $\mathrm{p} 20$, lasting for five days. The second purpose of this experiment was to determine if this learning was dependent on NMDA receptors. Therefore, within these subgroups, there was one group that received saline injections, and another that received an injection of the non-competitive NMDAr antagonist, MK-801. There were three age groups (starting at p16, p18, and p20) consisting of fourteen rats each; at each age group there were seven saline and seven MK-801-treated animals. Group 1 received water maze training from p16 to p20; Group 2 received water maze training from $\mathrm{p} 18$ to $\mathrm{p} 22$; and Group 3 received water maze training from $\mathrm{p} 20$ to $\mathrm{p} 24$. The same water maze protocol was used for this experiment as the previous, i.e. the modified water maze task.

Pups received a $0.1 \mathrm{ml}$ subcutaneous injection (SC) into the scruff of the neck of either $0.9 \%$ saline or $0.05 \mathrm{mg} / \mathrm{kg}$ MK-801 fifteen minutes prior to each day of juvenile water maze training. The MK-801 was made from a $1 \mathrm{mg} / \mathrm{ml}$ concentration and diluted with $0.9 \%$ saline. Previous research reported that the $0.05 \mathrm{mg} / \mathrm{kg}$ concentration led to significantly more lever pressing in the operant chamber than the $0.1 \mathrm{mg} / \mathrm{kg}$ concentration (Holahan et al., 2012). The higher concentration not only led to lower lever pressing, but lower overall activity levels by the rats as they had fewest nose pokes. Fewer nose pokes induced by the $0.1 \mathrm{mg} / \mathrm{kg}$ concentration of MK-801 suggests a general depressing effect 
on locomotor activities and impaired sensorimotor functions (Wozniak et al., 1990). The $0.05 \mathrm{mg} / \mathrm{kg}$ concentration is preferable, as it does not seem to impair the sensorimotor functions of the rat, therefore, it was believed that they would be capable to complete the water maze task.

\section{Experiment 3: Functional and structural changes in the hippocampus at developmental milestones}

There were six different groups for this experiment. Three groups of five animals were sacrificed on $\mathrm{p} 16, \mathrm{p} 18$, or $\mathrm{p} 20$. They received no sort of behavioural testing. Another three groups each with five animals underwent fixed platform water maze training for one day. The apparatus used was the juvenile water maze described above. There were eight trials conducted on one of three days; start points: p16, p18, p20. It took each animal approximately 20 minutes to complete all eight trials. Animals were returned to a cage that was equipped with a heating pad and waited there until they were sacrificed. Animals were sacrificed one hour after a one-day completion of water maze training to allow for c-Fos staining. The rats were placed into a carbon dioxide chamber then euthanized by decapitation.

\section{Experiment 4: spatial learning during juvenile period on a dry maze task}

This experiment was carried out to eliminate the performance issue of swimming ability in the water maze task. The decrease in latencies over age in the water maze could be due to performance improvements in the sense that the p20 rats are physically stronger and better at swimming than the p16 rats. The dry maze is a task that requires less physical labour and relies more solely on cognitive functions (spatial learning and memory). 
Rats were placed individually into the arena and allowed to explore. Two identical objects were placed in adjacent arena quadrants and remained in the same location across all familiarization phases. The familiarization period lasted seven minutes long and was carried out three times, once per hour over three hours. Rats were allowed to explore the objects for the full seven minutes. Between familiarization phases, the pups were placed back into their home cage with their litter mates. One and a half hours after the last familiarization phase, rats were tested for five minutes. The test consisted of one object being moved to a new quadrant that was adjacent to the old object. By moving one object to a new location for the testing phase, the procedure became identical to the conventional novel object-in-place (NOIP) preference test (Mumby et al., 2002; Gaskin et al, 2009). The NOIP task takes advantage of a rats spontaneous tendency to examine objects that have changed location within an otherwise stable environment more than they investigate objects that remain in constant locations (Dix \& Aggleton, 1999; Mumby et al., 2002; Gaskin et al., 2009). When rats display such a preference, one can infer that they have detected a change in the objects' location within the environment. To determine a rat's preference during the testing phase, an investigation ratio was calculated during the five minute testing phase; that is, the proportion of total object-investigation that was spent exploring the moved object $\left(t_{\text {moved }}\right.$ $\left./\left[\mathrm{t}_{\text {moved }}+\mathrm{t}_{\text {not moved }}\right]\right)$. A rat was considered to be investigating an object when its head was within $4 \mathrm{~cm}$ and oriented within $45^{\circ}$ of the object.

There were five groups of seven rats. Each group was trained and tested on a single day; p16, p17, p18, p19, or p20. The animals were only tested once to ensure that the preference for one object over another was based solely on the objects original 
positions during the familiarization phase. Testing over days would have led to the "contamination" of the memory and therefore preferences could not be accurately inferred, as the memories would begin to overlap with one another. The animals were given an intraperitoneal (IP) injection with sodium pentobarbital (1 mL euthanyl, 100 $\mathrm{mg} / \mathrm{mL}$ ) one hour after the test phase to allow for c-Fos staining, so they were no longer aware and then euthanized by decapitation.

\section{Immunohistochemistry}

Histology

For the third and fourth experiments, rats were euthanized one hour after the completion of whichever task they were assigned on different days (p16-p20) by decapitation. Brains were removed and fixed with 4\%-paraformaldehyde in $0.1 \mathrm{M}$ phosphate-buffered saline (PBS) overnight at $4^{\circ} \mathrm{C}$. This solution was replaced with $30 \%$ sucrose in $0.1 \mathrm{M}$ PBS the following day and brains were stored at $4^{\circ} \mathrm{C}$ until sectioned. Brains were hemisected and left hemispheres were sectioned at $60 \mu \mathrm{m}$ on a Leica CM1900 cryostat (Weztler, Germany). Sections from the left dorsal hippocampus were stored in a $0.1 \%$ sodium azide solution in $0.1 \mathrm{M}$ phosphate buffer $(\mathrm{PB})$ at $4^{\circ} \mathrm{C}$. $c-F o s$

For immunohistochemistry, sections were placed in Phosphate Buffered Saline with Triton X (T-PBS) for three, five-minute washes. They were then placed in their first block of $0.3 \%$ hydrogen peroxide $\left(\mathrm{H}_{2} \mathrm{O}_{2}\right)$ in T-PBS for 15 minutes, followed by three, five-minute washes in T-PBS. The second block was 3\% animal free blocker (AFB) in T-PBS for 30 minutes. Incubation in the primary antibody (rabbit anti-c-Fos from Abcam, 1:5000) occurred overnight at room temperature. The following day, tissue was 
washed in T-PBS for three, 10 minute washes followed by a two-hour incubation in the secondary antibody (anti-rabbit biotynilated from Vector Laboratories, 1:1000). Tissue was then rinsed for three, 10-minute washes in T-PBS before being placed into an $\mathrm{ABC}$ solution for one hour $(5 \mathrm{~mL}$ T-PBS, $75 \mu \mathrm{L}$ solution $\mathrm{A}, 75 \mu \mathrm{L}$ solution $\mathrm{B}$ - made at least 30 minutes before use). The tissue was rinsed with three, five-minute washes of Phosphate Buffered Saline (PBS) before being placed into the DAB solution (1mL DAB, $25 \mathrm{~mL}$ PBS, $625 \mu \mathrm{L} \mathrm{1 \%}$ cobalt chloride, $500 \mu \mathrm{L} 1 \%$ nickel ammonium sulfate and $1 \mathrm{~mL} \mathrm{3 \%}$ $\mathrm{H}_{2} \mathrm{O}_{2}$ ). Tissue was allowed to develop before being transferred into PBS and then mounted on glass slides. Slides were placed in a slide box where they dried overnight, safe from dust and other particles. Dehydration of the slides occurred the following day when the slides were completely dry. Slides were dipped into distilled water $\left(\mathrm{dH}_{2} \mathrm{O}\right)$ for one minute, $50 \%$ ethanol (EtOH) for one minute, followed by $95 \% \mathrm{EtOH}$ for five minutes, $100 \% \mathrm{EtOH}$ for 10 minutes and finally put into clearene for 20 minutes before being coverslipped with permount mounting medium.

C-Fos is a proto-ontogene in the immediate-early gene family. Immediate-early genes encode proteins and are induced by extracellular stimuli (Curran and Morgan, 1995). The c-fos protein can be identified with immunohistochemical techniques and is preferred to analyze its expression following stimulation (Herdegen et al., 1995). The basal expression of c-Fos is low in most structures, but it can be increased after various stimuli induction and is therefore a good marker for activated neurons (Herdegen et al., 1995). Herdegen and colleagues (1995) note that among other brain structures, there is some c-Fos immunolabelled neurons in the hippocampus, namely the CA1 and CA3. Therefore, the CA1 and CA3 will be the areas of interest when staining for c-Fos. 
c-Fos Quantification

For unbiased stereology, the CA1 and CA3 areas were traced at 10X magnification. Cell counts were performed at $60 \mathrm{X}$ magnification using parameters of $60 \mu \mathrm{m}$ by $60 \mu \mathrm{m}$ and an optical fractionator of $20 \mu \mathrm{m}$. Area of the traced sections were measured by planimetry and were analyzed to ensure no significant differences were found as the rats aged. Two sections from each rat were counted and the estimated total by mean measured thickness was analyzed. 


\section{Results}

\section{Experiment One}

Juvenile rats were trained on a modified spatial water maze task for nine days (p16-p24). On Day 1, rats were trained for eight trials during which the platform remained in the same location. On Day 2, the platform remained in the same location as the previous day for Trial 1 then was moved to a new location for Trials $2-8$. This pattern (same position on Trial 1 as the previous day's position for Trials 2 - 8 then moved to a new location) continued for the remaining training days.

Latencies to locate the platform were averaged over the eight trials to obtain a daily average for each animal. These data are plotted in Figure 2 and compared to data collected by Keeley and colleagues (2010) using the same procedure. 


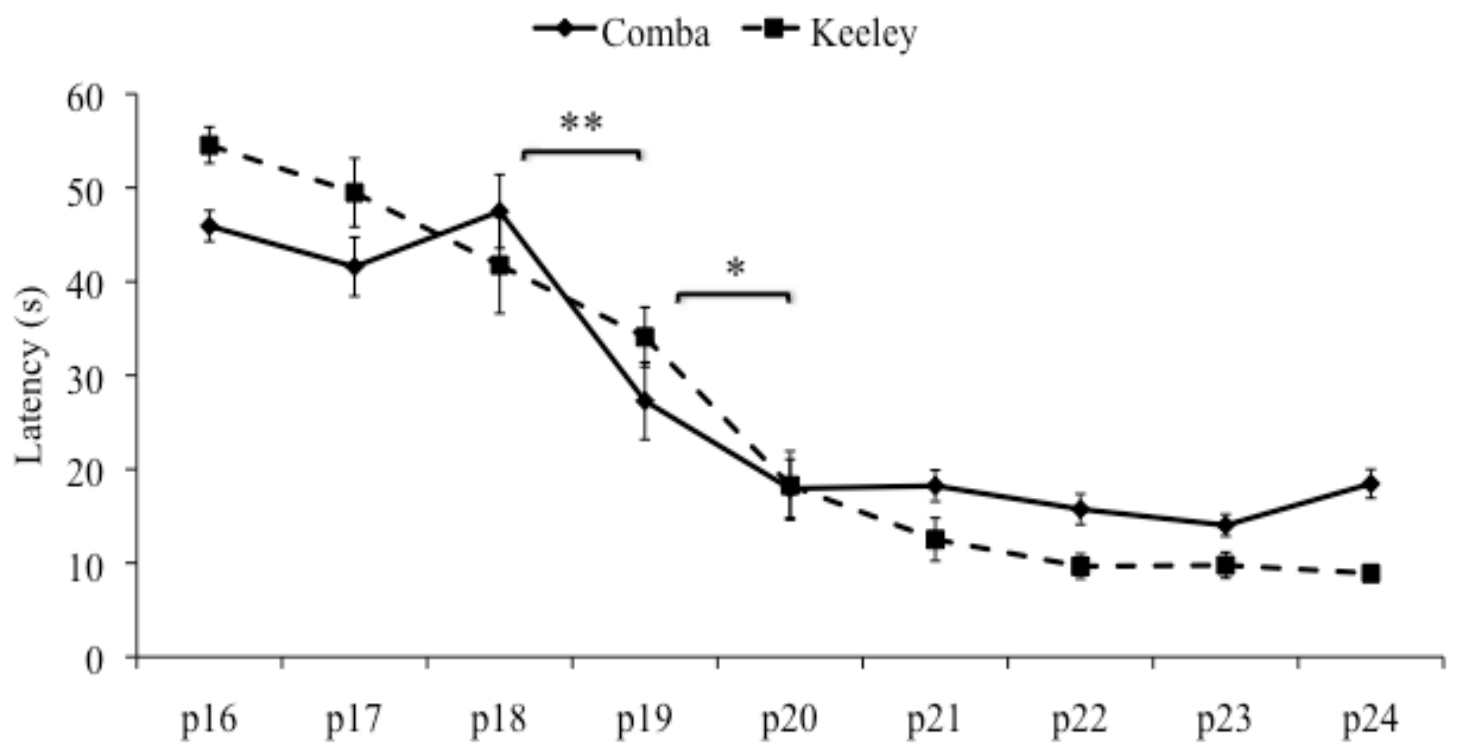

Figure 2. Latency to reach the hidden platform averaged across eight trials of daily training from p16 to p24 for current research (Comba) and previous research (Keeley). Significant differences were observed between p19 and p18 as well as p20 and p19. (*) $p<0.01 ;(* *) p<0.001$. 
The current data, as well as the previous data show that latencies decrease over days of training. A two-way repeated-measures ANOVA using day as the repeated measure and experimenter or group (Comba and Keeley) as between-subjects factors, revealed a main effect of day $\left(F_{(8,88)}=80.795, p<0.001\right)$ but no main effect of group $\left(F_{(1,11)}=0.165, p=0.692\right)$. To further examine the main effect of day and determine when latencies show significant changes over time, three $t$-tests were performed (p17 vs. p18; p18 vs. p19; p19 vs. p20) adjusting the alpha level using a Bonferroni correction $(0.05 / 3=0.017)$. Pairwise comparisons revealed no significant difference in latencies between $\mathrm{p} 17$ and $\mathrm{p} 18($ difference $=0.38 \pm 3.81$; mean $\pm \operatorname{SEM} p=0.92)$. Pairwise comparisons did reveal significant differences in latencies between p18 and p19 (14.41 \pm $3.23 p<0.001)$ and between p19 and p20 $(12.34 \pm 3.43 p<0.01)$. The largest decrease in latencies over days occurred from $\mathrm{p} 18$ to $\mathrm{p} 19$ and again from $\mathrm{p} 19$ to $\mathrm{p} 20$. The dramatic decrease in time it takes to reach the hidden platform during this period (p16-p20) suggests spatial learning abilities may be emerging in this specific stage of development.

\section{Experiment Two}

Juvenile rats were trained on a modified spatial water maze task for five days. There were three different start dates used for this experiment: Group 1 began on p16, Group 2 began on p18, and Group 3 began on p20. Within each group, seven animals received a saline injection and the remaining seven received a non-competitive NMDA receptor antagonist, MK-801 injection fifteen minutes prior to training. The modified spatial water maze task was carried out in the same fashion as Experiment one. 


\section{Group 1 - postnatal day 16-20}

Latency data plotted in Figure 3a show that latencies in the saline group decreased more so over days than latencies in the MK-801 group. A two-way repeated-measures ANOVA (drug treatment as the between-subjects factor and day as the repeated measure) revealed main effects of drug group $\left(F_{(1,12)}=10.841, p<0.01\right)$ and day $\left(F_{(4,48)}=11.032, p\right.$ $<0.001)$ and a significant interaction between these factors $\left(F_{(4,48)}=2.905, p<0.05\right)$. To further examine the interaction, three independent-sample $t$-tests were performed (p18, p19 and p20). Significant differences were seen at p18 $(t(12)=2.297, p<0.05), \mathrm{p} 19$ $(t(12)=3.132, p<0.01)$ and $\mathrm{p} 20(t(12)=3.154, p<0.01)$. The main effect of drug group provides evidence that the saline rats outperformed the rats given the NMDA receptor antagonist.

A key element in successfully completing the water maze task is the ability to swim. The maze is physically taxing, as it requires swimming to find the hidden platform. Therefore speed, a performance factor, was analyzed to examine if rats under the influence of MK-801 displayed any performance deficits that would overshadow learning impairments. These data are plotted in Figure 3b. A two-way repeatedmeasures ANOVA (drug condition as the between factor and day as the repeated measure) revealed main effects of $\operatorname{drug}$ group $\left(F_{(1,12)}=44.719, p<0.001\right)$ and day $\left(F_{(4,48)}\right.$ $=14.086, p<0.001)$, but no significant interaction $\left(F_{(4,48)}=2.307, p=0.072\right)$. These data show that swimming speed increases with age and rats under the influence of MK801 swim slower than saline-injected rats. 

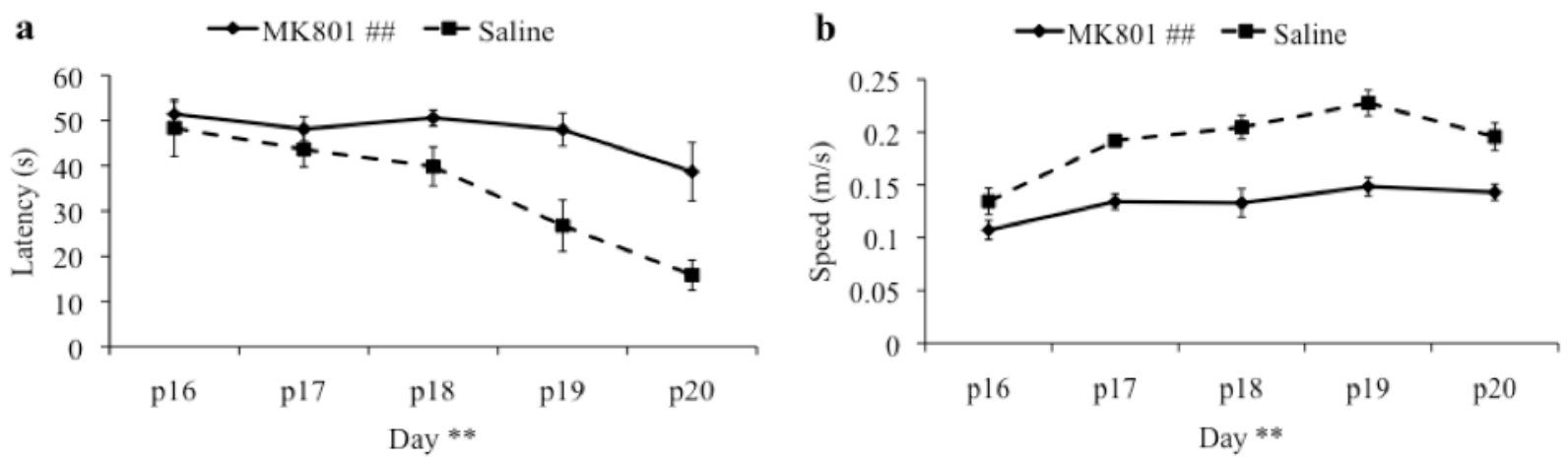

Figure 3. (a) Latency to reach the hidden platform averaged across eight trials of daily training for rats treated with MK-801 or saline from p16 to p20 and (b) the speed averaged over eight trials of daily training. (\#\#) Indicates a main effect of group $(p<0.001) ;(* *)$ indicates a main effect of day $(p<$ $0.001)$. 
Group 2 - postnatal day $18-22$

Latency data plotted in Figure 4a revealed that latencies decreased over days in both MK-801 and saline groups. A two-way repeated-measures ANOVA revealed main effects of drug group $\left(F_{(1,12)}=6.787, p<0.05\right)$ and day $\left(F_{(4,48)}=8.623, p<0.001\right)$ but no significant interaction between these factors $\left(F_{(4,48)}=0.386, p=0.818\right)$. Overall, the saline rats outperformed the MK-801 treated rats.

Speed data are plotted in Figure 4b. A two-way repeated-measures ANOVA revealed a main effect of day $\left(F_{(4,48)}=6.007, p=0.01\right)$ but no main effect of drug group $\left(F_{(1,12)}=3.836, p=0.074\right)$ and no significant interaction $\left(F_{(4,48)}=0.715, p=0.586\right)$. Although the saline group had significantly shorter latencies to reach the hidden platform than the MK-801 group, the drug groups did not differ on speed. 

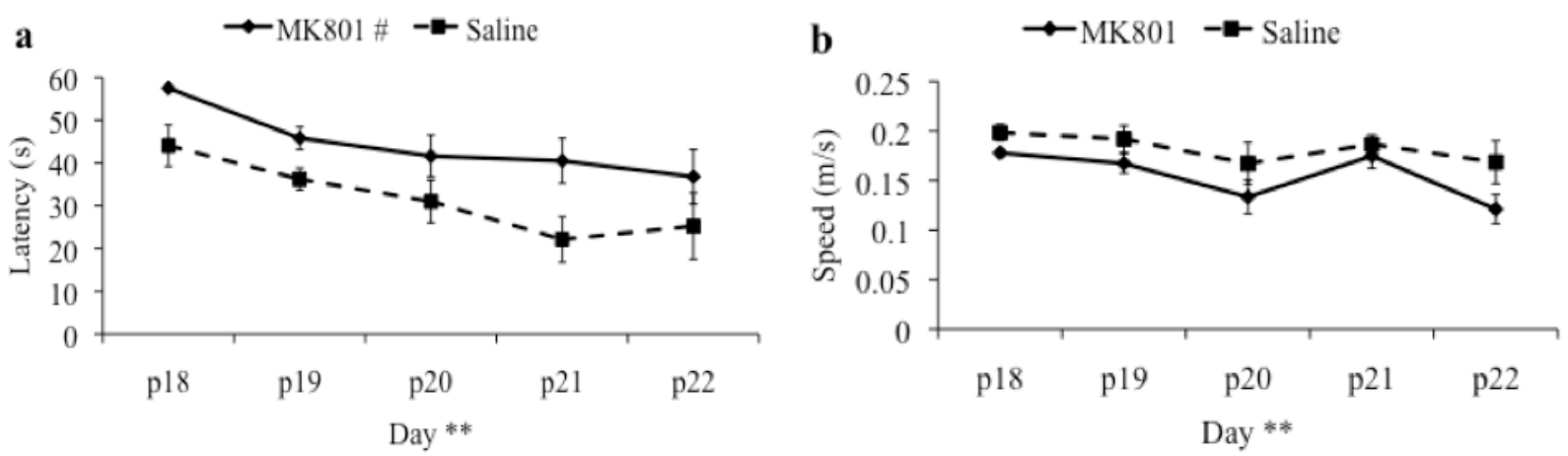

Figure 4. (a) Latency to reach the hidden platform averaged across eight trials of daily training for rats treated with MK-801 or saline from p18 to p22 and (b) the speed averaged over eight trials of daily training.

(\#) Indicates a main effect of group, $(p<0.05) ;(* *)$ indicates a main effect of day $(p<$ $0.001)$. 
Group 3 - postnatal day 20-24

Latency data plotted in Figure 5a revealed a decrease in latencies to locate the platform in both the saline and MK-801 groups over the five days of training. A two-way repeated-measures ANOVA revealed main effects of drug group $\left(F_{(1,12)}=6.318, p<\right.$ $0.05)$ and day $\left(F_{(4,48)}=13.396, p<0.001\right)$, but no significant interaction between these two factors $\left(F_{(4,48)}=1.335, p=0.271\right)$. Therefore, the saline group had significantly shorter latencies overall to find the hidden platform than the MK-801 group.

Speed data plotted in Figure $5 \mathrm{~b}$ revealed that speed increased over days but the MK-801 group seemed to swim slower than the saline group. A two-way repeatedmeasures ANOVA revealed main effects of drug group $\left(F_{(1,12)}=8.048, p<0.05\right)$ and day $\left(F_{(4,48)}=9.046, p<0.001\right)$, but no significant interaction between these factors $\left(F_{(4,48)}=\right.$ 1.303, $p=0.282$ ). The swim speeds in rats injected with MK-801were slower than the saline group and hence, the longer latencies following MK-801 injections may be explained by their slower swim speeds. 

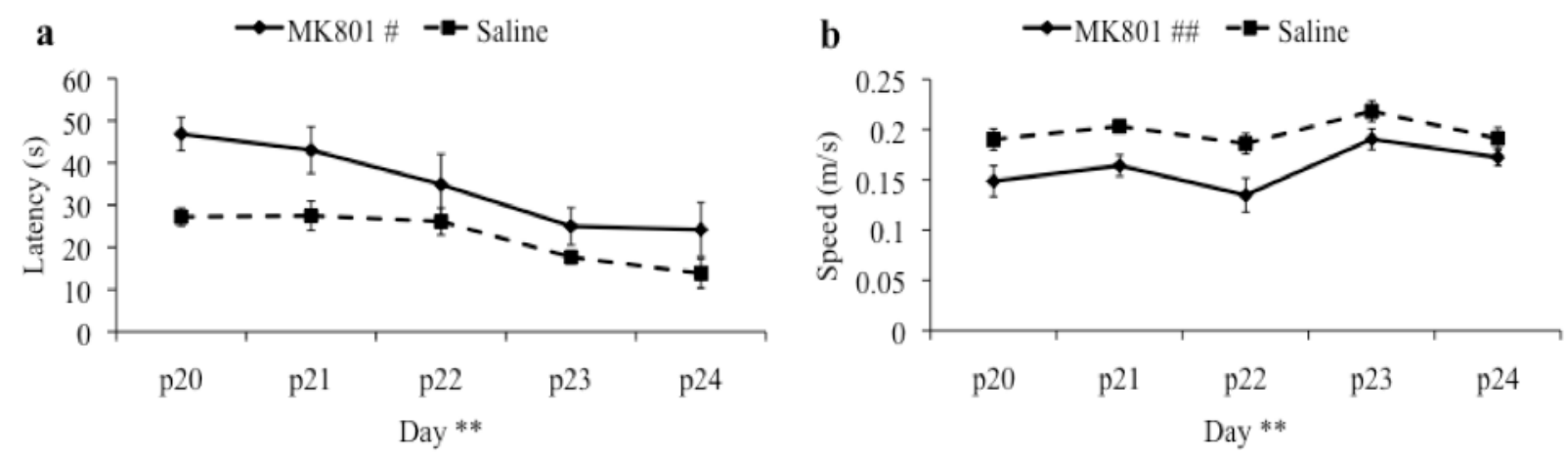

Figure 5. (a) Latency to reach the hidden platform averaged across eight trials of daily training for rats treated with MK801 or saline from p20 to p24 and (b) the speed averaged over eight trials of daily training.

(\#) Indicates a main effect of group $(p<0.05)$; (\#\#) indicates a main effect of group $(p<$ $0.001) ;(* *)$ indicates a main effect of day $(p<0.001)$. 
All groups

It is apparent from these data that the saline rats learn well over their five days of training regardless of what days they received training. However, it was of interest to determine whether these rats all began at the same level and showed similar improvements on the task over time, or whether the older rats were superior to the younger rats. The data from the individual start dates $(\mathrm{p} 16, \mathrm{p} 18, \mathrm{p} 20)$ are plotted in Figure 6. A one-way ANOVA on Day 1 latencies for each group revealed a main effect of group $\left(F_{(2,20)}=5.596, p<0.05\right)$. Tukey post-hoc tests revealed significant differences between $\mathrm{p} 20$ and $\mathrm{p} 16(p<0.05)$ and near-significant differences between $\mathrm{p} 20$ and p18 ( $p$ $=0.053)$. Therefore, there was a decrease in latencies on the first day of training with no prior learning. This suggests that age is a factor in the ability to undergo spatial learning on the water maze task and that this ability emerges around p20. 


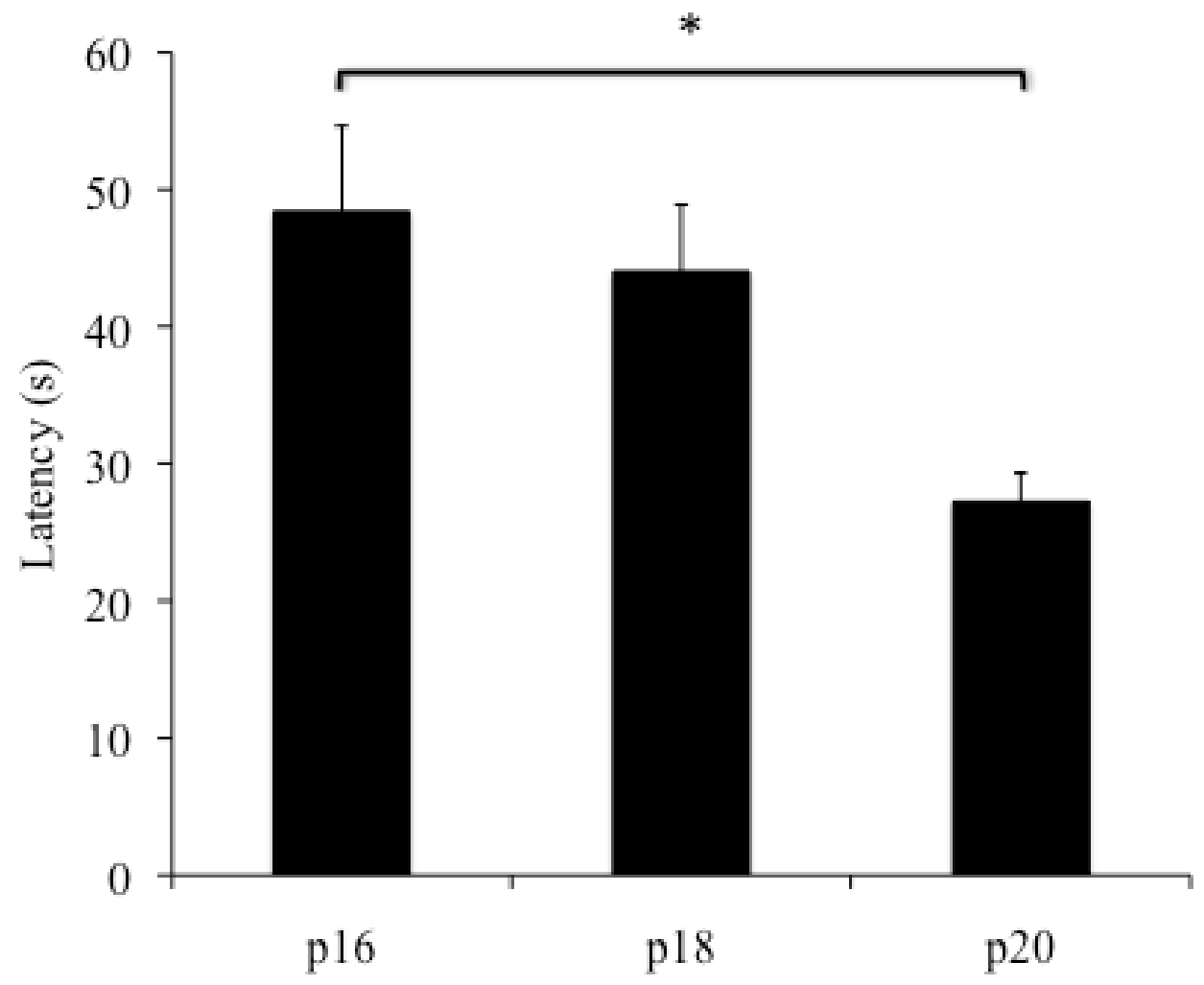

Figure 6. Latency to reach the hidden platform averaged across eight trials for each groups start date: p16, p18 or p20.

(*) Indicates significant group differences between p20 and p16 $(p<0.05)$. 


\section{Experiment Three}

Three groups of five rats underwent water maze training for eight trials on one day only: p16, p18, or p20 then were sacrificed. Another three groups of five rats each received no water maze training and were sacrificed on p16, p18, or p20.

\section{Behavioural Results}

Those that underwent water maze training were analyzed for latency (see Figure 7) and speed (see Figure 8) measures. A two-way repeated-measures ANOVA on latency data revealed a main effect of group $\left(F_{(2,12)}=8.490, p<0.01\right)$ and a main effect of trial $\left(F_{(7,84)}=2.443, p<0.05\right)$, but no significant interaction $\left(F_{(14,84)}=0.754, p=0.714\right)$. Tukey post-hoc tests revealed significant differences between p20 and p16 $(p<0.01)$ as well as p20 and p18 $(p<0.05)$. Therefore, the emergence of spatial ability on the water maze seems to appear at $\mathrm{p} 20$.

It was of interest to decipher whether these differences were due to performance factors (i.e. the rats are stronger at an older age and better able to perform the task) or functional changes in the brain (i.e. the older rats are undergoing changes in the brain which allow for this level of spatial learning). One way to examine this is to look at speed as a performance factor. A two-way repeated-measures ANOVA on speed revealed a main effect of group $\left(F_{(2,12)}=85.195, p<0.001\right)$ but no main effect of trial $\left(F_{(7,84)}=0.968, p=0.460\right)$ and no significant interaction $\left(F_{(14,84)}=1.425, p=0.160\right)$. Tukey post-hoc tests revealed significant differences between p20 and p16 $(p<0.001)$ as well as p20 and p18 $(p<0.001)$. Therefore, the older rats were able to swim faster which may contribute to their improved performance on the water maze. 


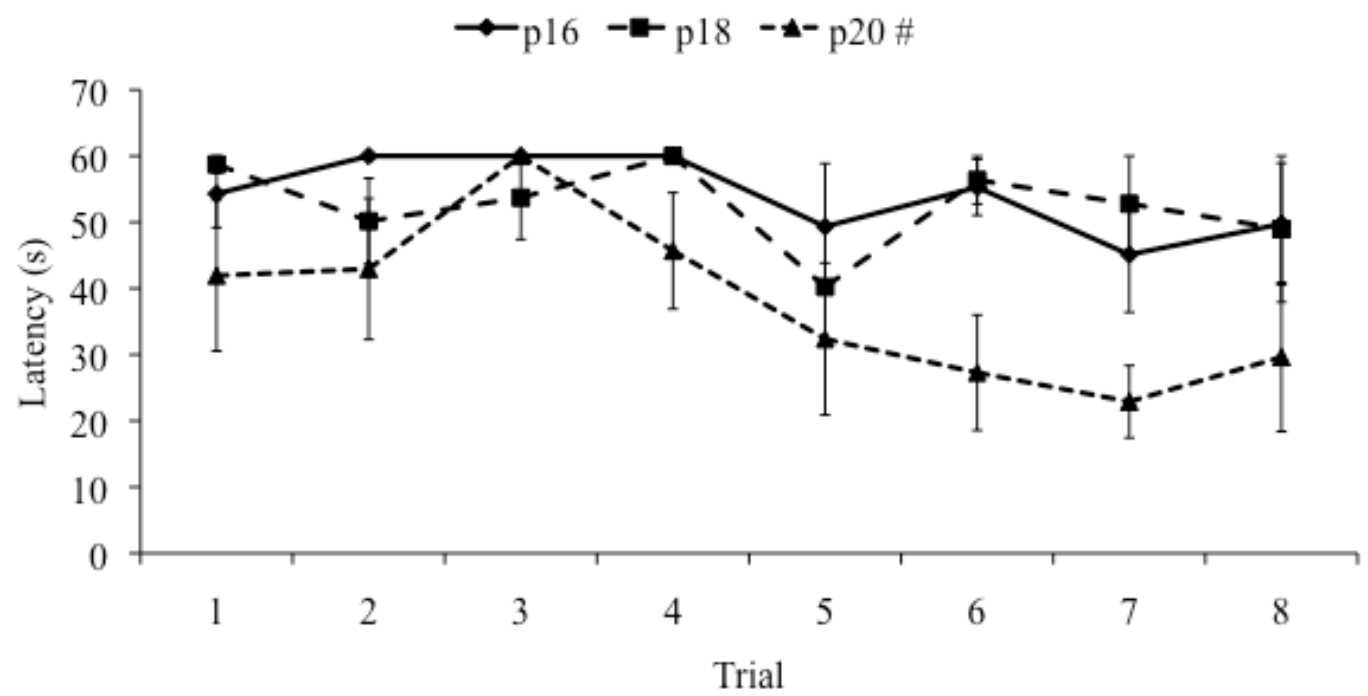

Figure 7. Latency to reach the hidden platform for one day only at start dates: p16, p18 and $\mathrm{p} 20$.

(\#) Indicates significant group differences $(p<0.01)$. Tukey post-hoc tests reveal p20 is significantly different from p18 $(p<0.05)$ and from p16 $(p<0.01)$. 


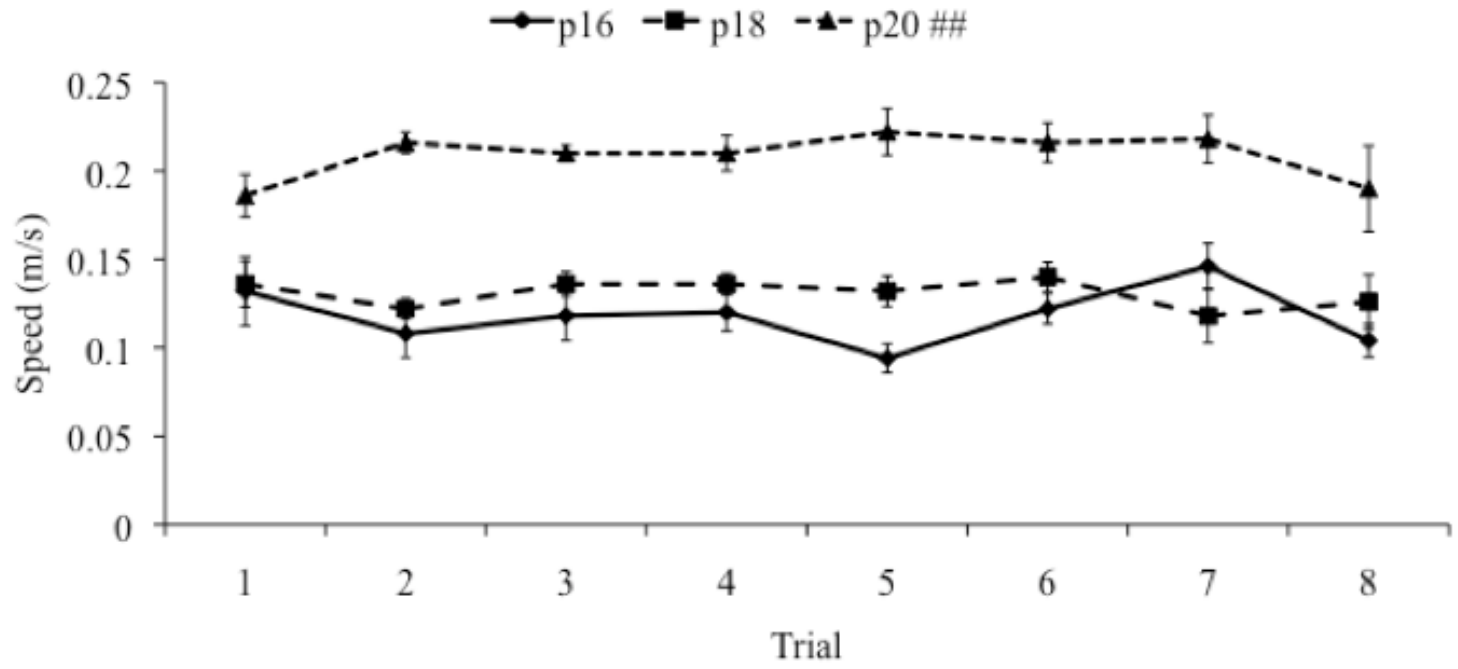

Figure 8. Swim speed of the rats to reach the hidden platform for one day only at start dates: p16, p18 and p20.

(\#\#) Indicates significant group differences $(p<0.001)$. Tukey post-hoc tests reveal p20 is significantly different from $\mathrm{p} 18(p<0.01)$ and from $\mathrm{p} 16(p<0.01)$. 


\section{Immunohistochemistry}

In order to examine whether there were functional neural circuit changes occurring during this developmental period, immunohistochemical techniques were used to label c-Fos immunopositive cells in two regions of the hippocampus: CA1 and CA3.

\section{Immunohistochemical Results}

\section{$C A 3$}

Mean cell count for the CA3 region of the hippocampus is plotted in Figure 9. A two-way ANOVA revealed main effects of age $\left(F_{(2,24)}=17.057, p<0.01\right)$ and training $\left(F_{(1,24)}=16.292, p<0.01\right)$ but no significant interaction $\left(F_{(2,24)}=2.399, p=0.112\right)$. Those with training had significantly more c-Fos immunopositive cells in the CA3 region of the hippocampus. Tukey post-hoc tests on age revealed significant differences between p20 and p16 $(p<0.001)$ as well as p20 and p18 $(p=0.001)$ showing more activation in the CA3 at an older age and after water maze training.

Figure 10 shows representative images from all three age groups and both treatment groups.

To account for size of the hippocampus growing as the rats mature, the counted CA3 area was traced and the areas were analyzed (Fig 11). A two-way ANOVA revealed no main effects of age $\left(F_{(2,24)}=1.871, p=0.176\right)$ or training $\left(F_{(1,24)}=2.367, p=0.137\right)$. 


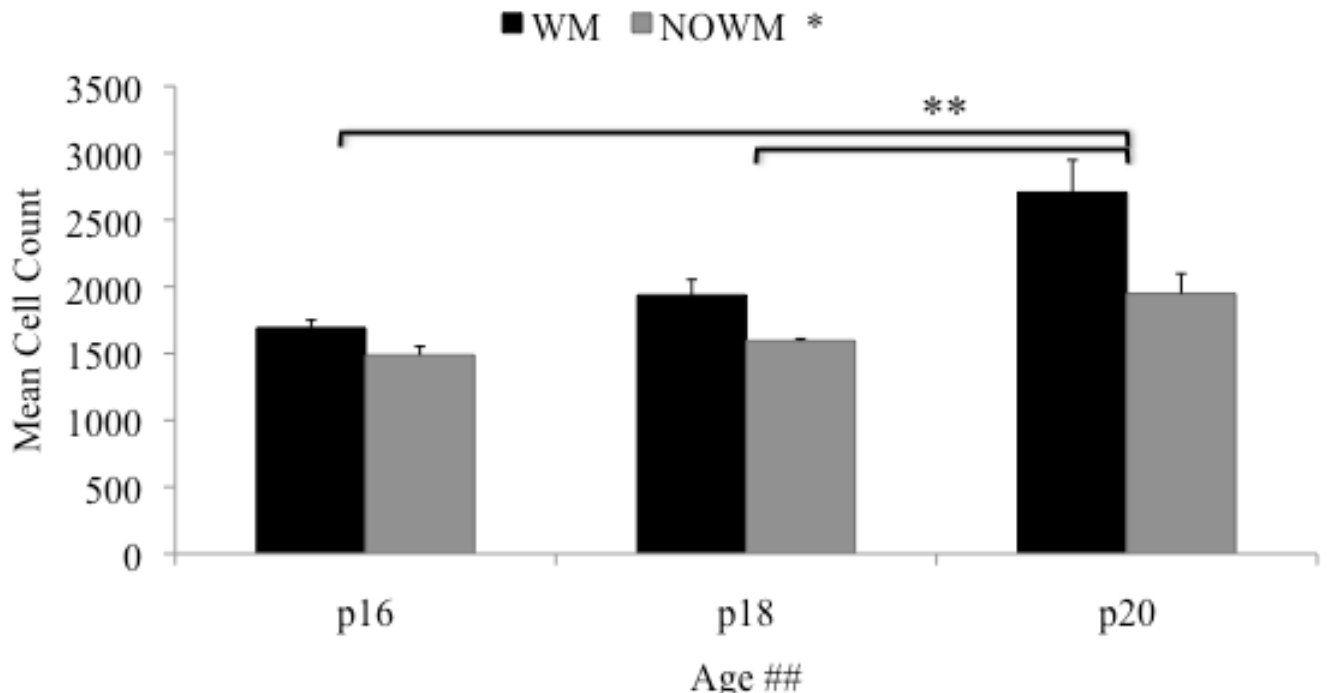

Figure 9. Quantification of c-Fos staining in the CA3 region of the hippocampus with and without water maze training for: $16, \mathrm{p} 18$ and p20.

(\#\#) Indicates a main effect of age $(p<0.01) ;(*)$ indicates a main effect of training $(p<$ $0.01) ;(* *)$ indicates significant differences using Tukey post-hoc tests between p20 and p18 as well as p20 and p16 $(p<0.001)$. 
CA3
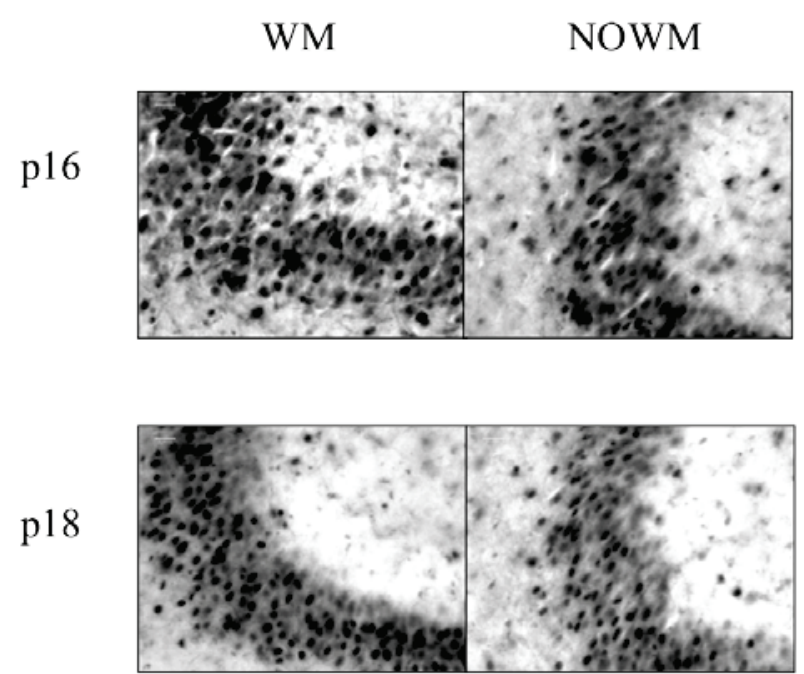

p18

p20

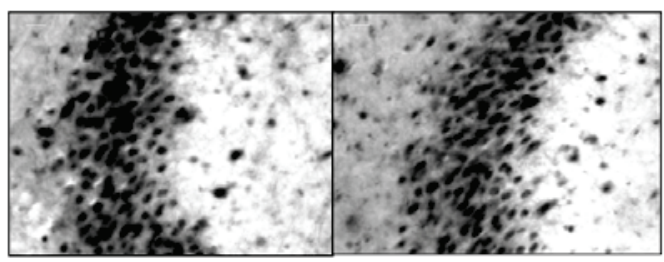

CA1
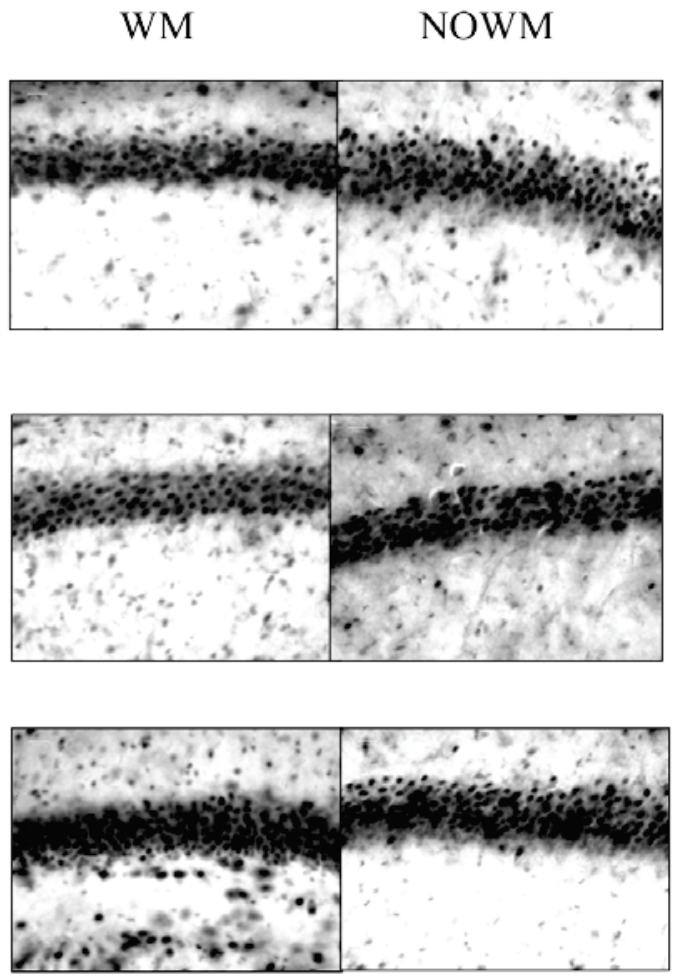

Figure 10. Representative images for c-Fos immunopositive cells in the CA3 and CA1 regions of the hippocampus for rats with (WM) or without (NOWM) water maze training. Images were taken at $40 \mathrm{X}$ with scale bar $=20 \mu \mathrm{M}$. 


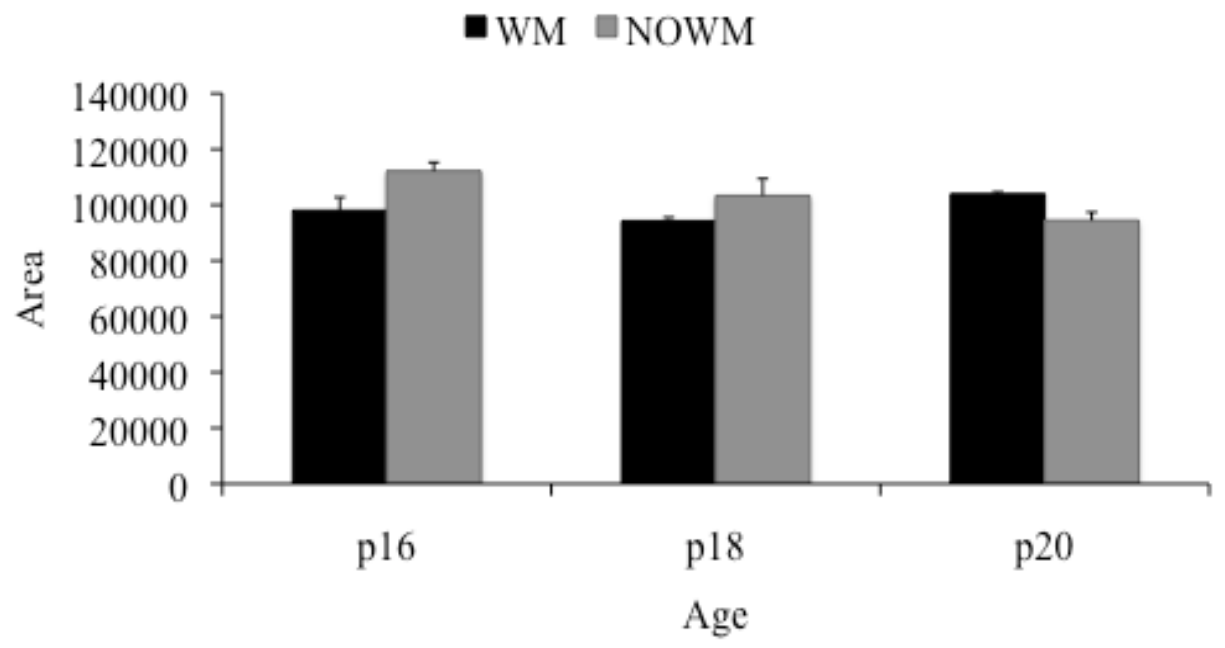

Figure 11. Area of the CA3 region of the hippocampus was traced and no main effects of age or training were found. 


\section{CA1}

Mean cell count for the CA1 region of the hippocampus is plotted in Figure 12. A two-way ANOVA revealed a main effect of age $\left(F_{(2,24)}=12.828, p<0.001\right)$, but no main effect of training $\left(F_{(1,24)}=0.001, p=0.982\right)$ and no significant interaction $\left(F_{(2,24)}=\right.$ $0.556, p=0.581)$. Tukey post-hoc tests on age revealed significant differences between $\mathrm{p} 20$ and $\mathrm{p} 16(p<0.001)$ as well as p20 and p18 $(p=0.001)$. Thus, age is a factor in the number of c-Fos positive neurons in the CA1 region and the increase seen in p20 rats occurred whether or not the rat underwent water maze training.

Figure 10 shows representative images from all three age groups and both treatment groups.

To account for size of the hippocampus growing as the rats mature, the area of the CA1 was traced and the areas were analyzed (Fig 13). A two-way ANOVA revealed no main effects of age $\left(F_{(2,24)}=2.215, p=0.131\right)$ or training $\left(F_{(1,24)}=0.895, p=0.354\right)$. 


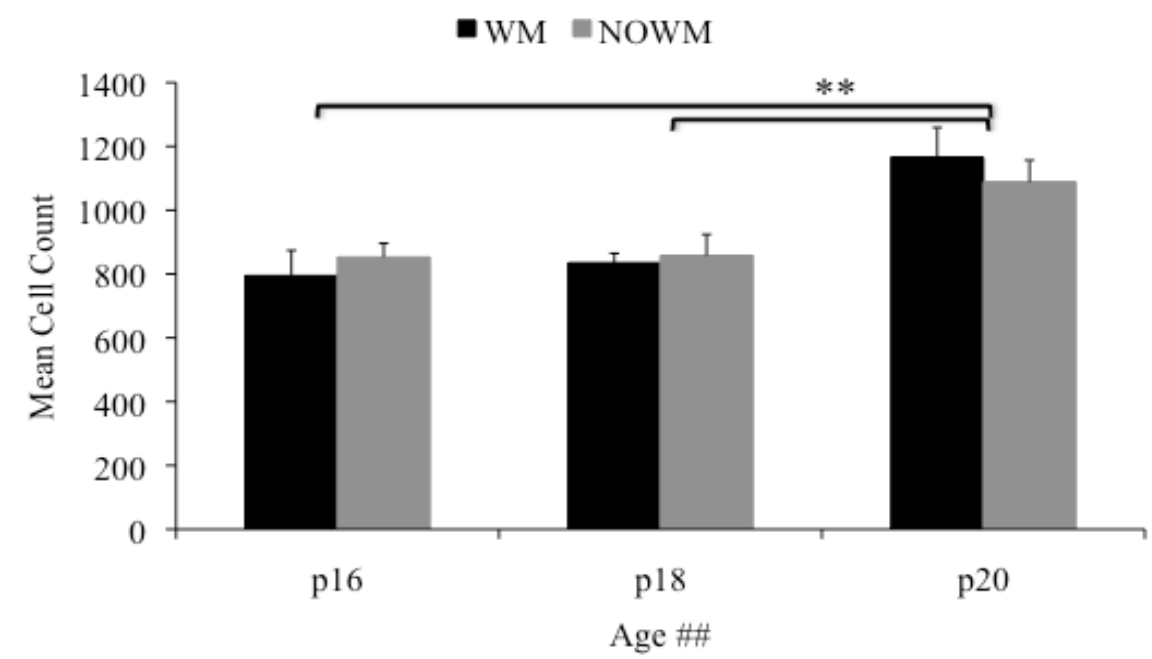

Figure 12. Quantification of c-Fos positive cells in the CA1 region of the hippocampus with and without water maze training at: p16, p18 and p20.

(\#\#) Indicates a main effect of age $(p<0.001) ;(* *)$ indicates significant differences using Tukey post-hoc tests between $\mathrm{p} 20$ and p18 as well as p20 and p16 $(p</=0.001)$. 


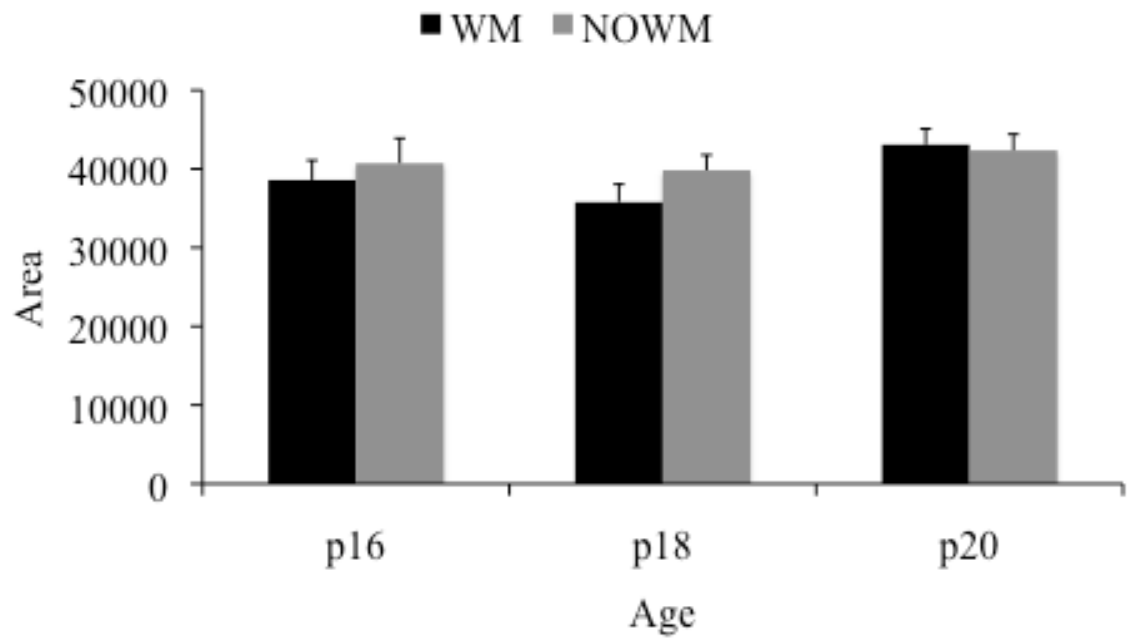

Figure 13. Area of the CA1 region of the hippocampus was traced and no main effects of age or training were found. 


\section{Experiment Four}

In order to examine whether the differences in latencies seen in Experiment 3 were due to performance factors (speed) or cognitive function as reflected in c-Fos labelling seen in the hippocampus, a fourth experiment was carried out on a less physically demanding, dry maze task.

Behavioural Results

The mean investigation ratios are plotted in Figure 14. Only two age groups showed mean investigation ratios that were significantly above chance, p19 $(t(6)=2.768$, $p<0.05)$ and $\mathrm{p} 20(t(6)=2.517, p<0.05)$. 


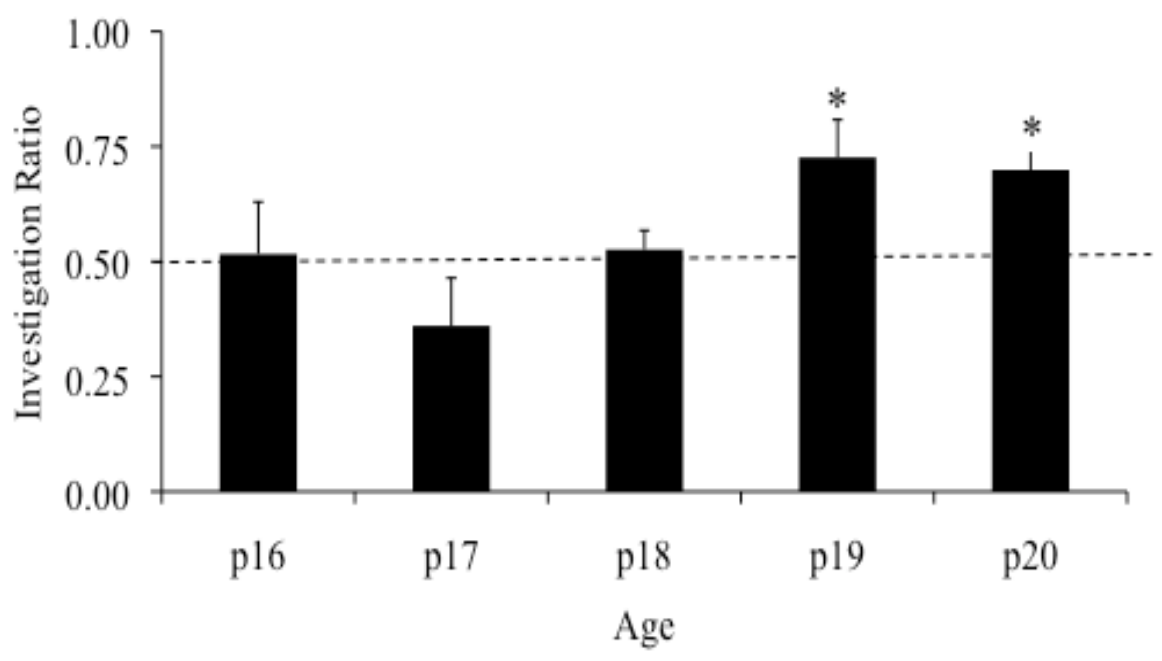

Figure 14. Investigation ratios from the five minute test period in the dry maze task for the five age groups: p16, p17, p18, p19 and p20.

(*) Indicates the p19 and p20 investigation ratios are significantly higher than chance $(p<$ $0.05)$. 


\section{Immunohistochemical Results}

\section{$C A 3$}

Mean cell count for the CA3 region of the hippocampus is plotted in Figure 15. A one-way ANOVA revealed significant group differences $\left(F_{(4,30)}=2.879, p<0.05\right)$.

Tukey post-hoc tests revealed significant differences between $\mathrm{p} 20$ and $\mathrm{p} 16(p<0.05)$.

Figure 16 shows representative images from all five age groups.

To account for size of the hippocampus growing as the rats mature, the counted CA3 area was traced and the areas were analyzed (Fig 17). A one-way ANOVA revealed no significant group differences $\left(F_{(4,30)}=0.489, p=0.744\right)$. 


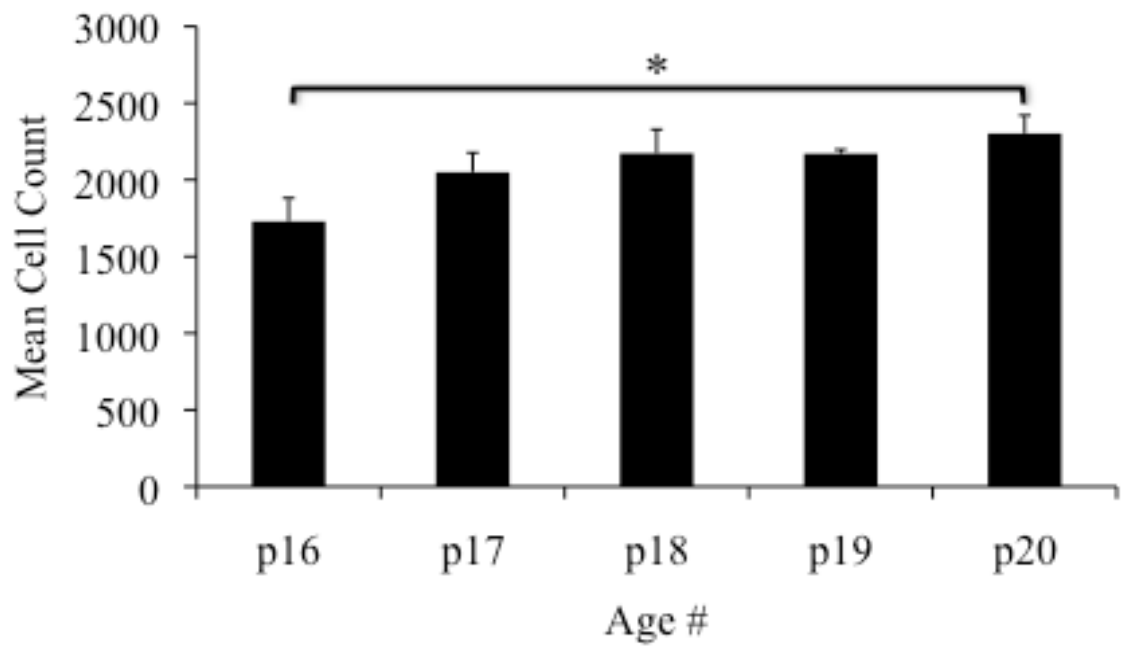

Figure 15. Quantification of c-Fos staining in the CA3 region of the hippocampus following dry maze training and testing at: p16, p17, p18, p19 or p20.

(\#) Indicates a main effect of age $(p<0.05) ;(*)$ indicates a significant difference using Tukey post-hoc tests between p20 and p16 $(p<0.05)$. 


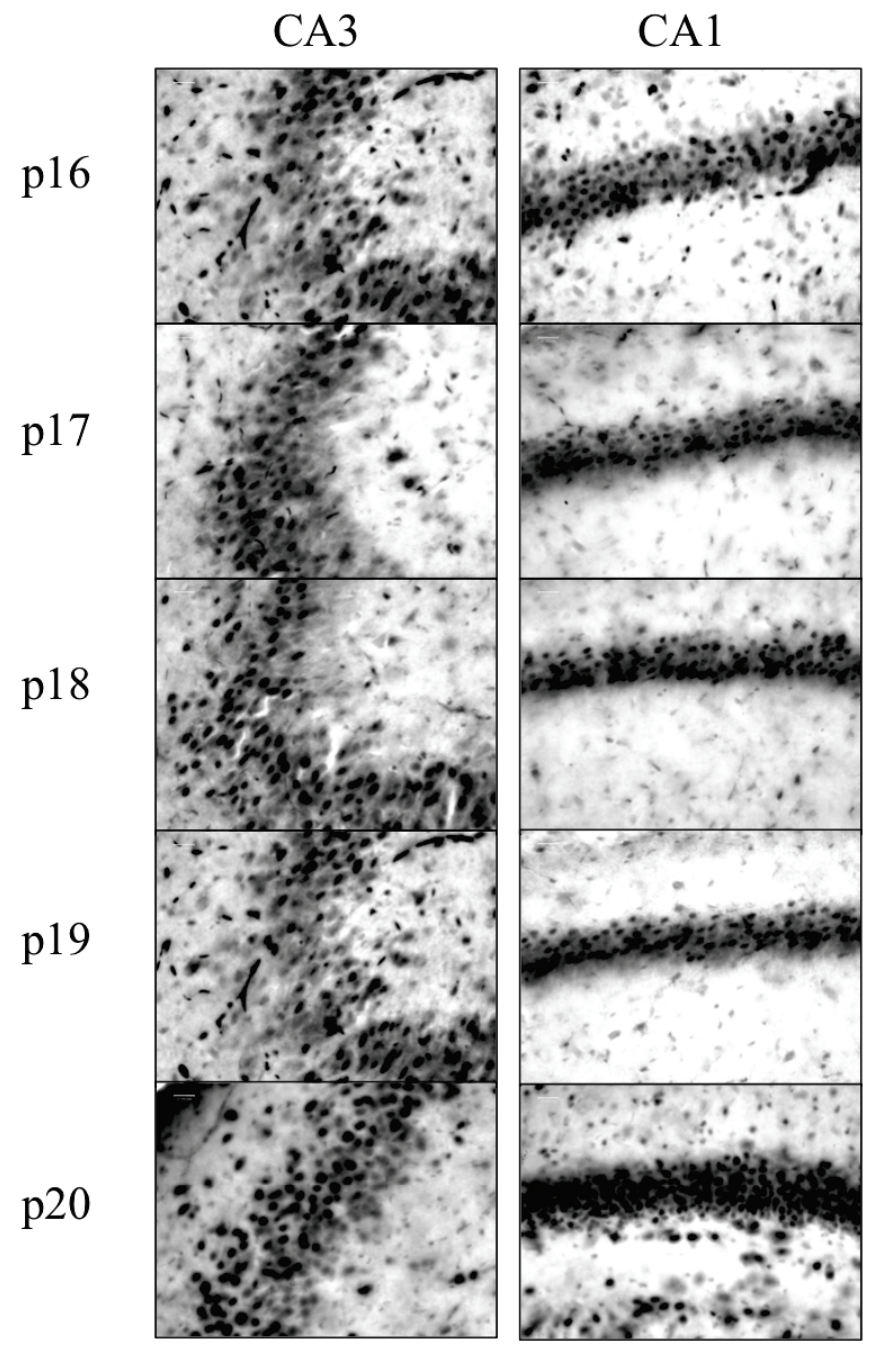

Figure 16. Representative images for c-Fos immunopositive cells in the CA3 and CA1 regions of the hippocampus for rats following dry maze training.

Images were taken at $40 \mathrm{X}$ with scale bar $=20 \mu \mathrm{M}$. 


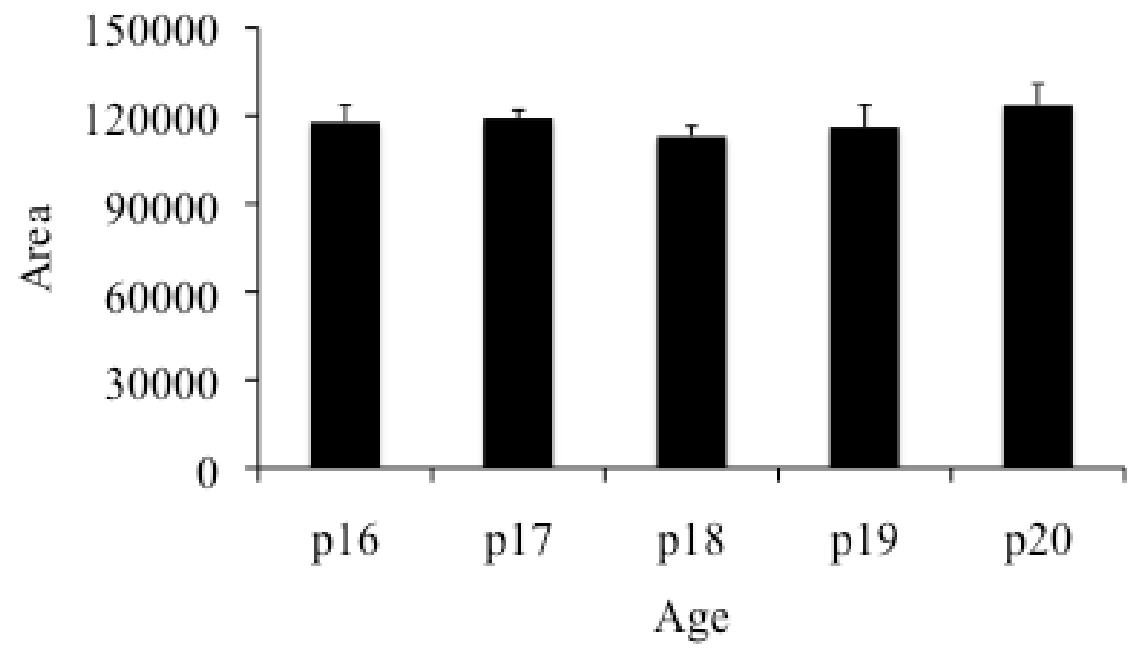

Figure 17. Area of the CA3 region of the hippocampus was traced and no main effect of age was found. 


\section{CA1}

Mean cell count for the CA3 region of the hippocampus is plotted in Figure 18. A one-way ANOVA revealed significant group differences $\left(F_{(4,30)}=14.272, p<0.001\right)$. Tukey post-hoc tests revealed significant differences between p20 and all other ages; p20 and $\mathrm{p} 19(p<0.05), \mathrm{p} 20$ and $\mathrm{p} 18(p<0.001), \mathrm{p} 20$ and $\mathrm{p} 17(p<0.001), \mathrm{p} 20$ and $\mathrm{p} 16(p<$ 0.001). The Tukey post hoc tests also revealed a significant difference between p19 and $\mathrm{p} 17(p<0.05)$

Figure 16 shows representative images from all five groups.

To account for size of the hippocampus growing as the rats mature, the counted CA1 area was traced and analyzed (Fig 19). A one-way ANOVA revealed no significant group differences $\left(F_{(4,30)}=1.024, p=0.411\right)$. 


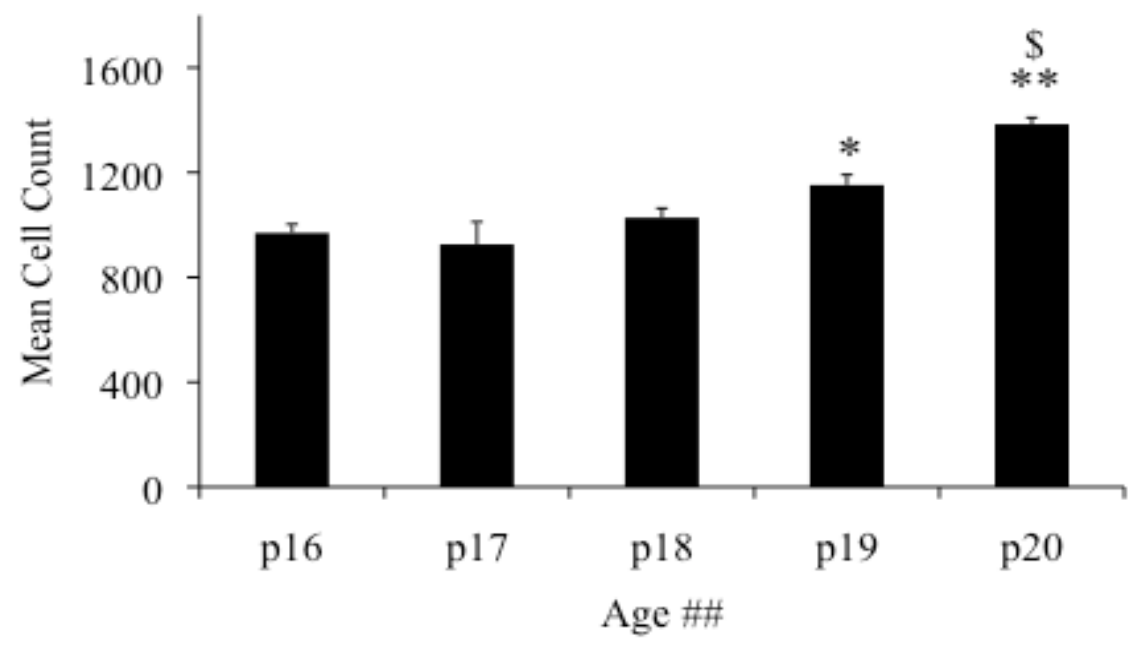

Figure 18. Quantification of c-Fos staining in the CA1 region of the hippocampus following dry maze training and testing at: p16, p17, p18, p19 or p20.

(\#\#) Indicates a main effect of age $(p<0.001) ;(*)$ indicates a significant difference using Tukey post-hoc tests between p19 and p17 $(p<0.05) ;(* *)$ indicates a significant difference between p20 and all other ages $(p<0.001)$; $(\$)$ indicates a significant difference between p20 and p19 $(p<0.05)$. 


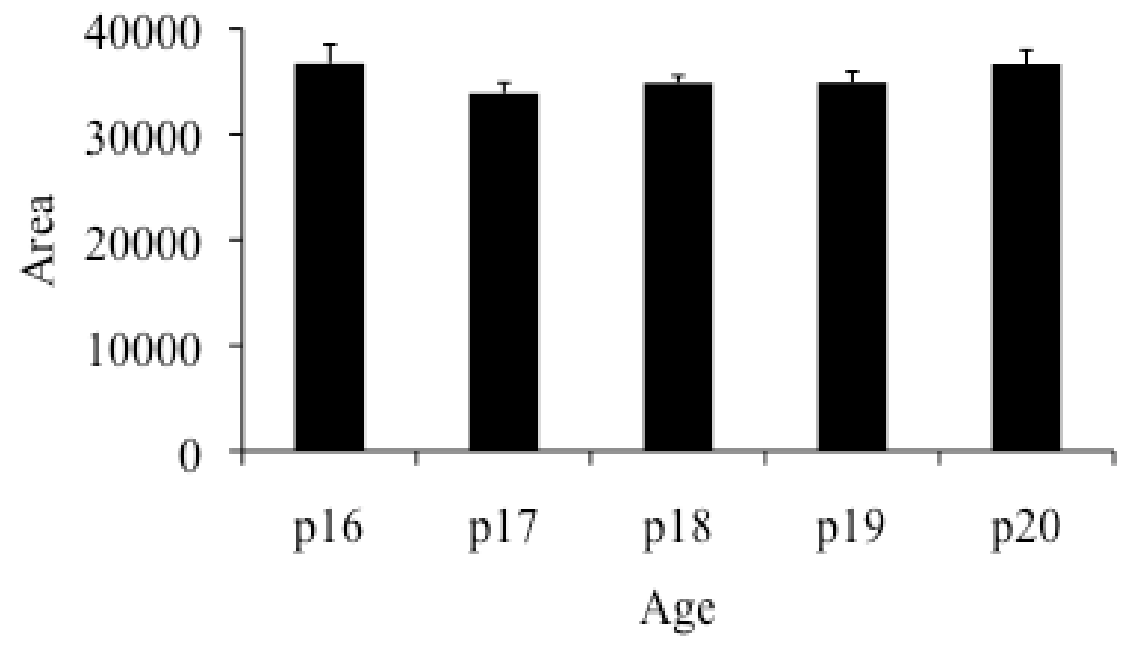

Figure 19. Area of the CA1 region of the hippocampus was traced and no main effect of age was found. 


\section{Discussion}

\section{Summary of results}

In Experiment 1, from p16 to p18, rats had similar latencies to reach the hidden platform and did not show much improvement over these days. From p18 to p20, the rats showed a significant decrease in latencies to find the platform suggesting spatial learning was occurring. By $\mathrm{p} 20$, the rats performed the task well and could find the hidden platform quickly showing asymptotic performance. These results replicate those of Keeley and colleagues (2010) and Wartman and colleagues (2012).

In Experiment 2, Group 1 (p16 to p20) also showed minimal learning from p16 to p18 for both treatment and control groups. From p18 onwards, a decrease in latency occurred for the saline-treated group and they performed very well by p20. For Group 2 (p18 to p22) the saline-treated rats performed better than the MK- 801 treated rats and they also, similar to Group 1, performed well by p20. Lastly, Group 3 (p20 to p24) salinetreated rats started off much faster than the MK-801-treated rats. The latencies on the

first day of training in Group 3 (p20) were significantly lower than the first day in Group 1 (p16), suggesting a possible emergence of spatial function independent from practice and any prior experience with the water maze.

Experiment 3 examined a one-day training procedure and subsequent c-Fos staining in the CA1 and CA3 regions of the hippocampus. The group given eight trials on p20 had significantly lower latencies than rats trained on p16 or rats trained on p18. The group trained on p20 also showed higher numbers of c-Fos immunopositive cells in the $\mathrm{CA} 1$ and $\mathrm{CA} 3$ regions of the hippocampus indicating more activity in these regions at p20. However, the p20 group also showed significantly faster swim speeds. This raises 
the possibility that the older rats were better able to perform the task than p16 and p18 rats because they were stronger and therefore better swimmers. This suggests that physicality may have been a contributing factor for improvement on the water maze task making it difficult to discern the role of cognition for successful completion of the water maze task. Because swimming is more demanding in terms of physical aptitude, an older rat may show better performance because they have more muscle mass to perform the task. Therefore, using a task with less physical demands may help dissociate between cognitive and physical factors.

To examine whether the improvements seen in the water maze were due to cognitive or motor development, a final experiment was run on a dry maze. This task was less physically demanding (exploration rather than swimming) and thus was hypothesized to rely more on cognitive functions. The results from Experiment 4 revealed that p19 and p20 might indeed be a critical time period for spatial development. The rats performed significantly better than chance at $\mathrm{p} 19$ and $\mathrm{p} 20$ but younger rats $(\mathrm{p} 16$, p17, p18) showed chance performance. Similar improvements seen on a less physically demanding task suggest the emergence of spatial-cognitive functioning around this age rather than simply ascribing the improved learning to increases in physicality or motor development. Rats at p20 also showed significantly more c-Fos immunopositive cells in the $\mathrm{CA} 1$ and $\mathrm{CA} 3$ regions of the hippocampus suggesting that these regions are more active as the rat ages.

One preliminary conclusion to draw from the behavioural data of all four experiments is that p20 is a critical age for the emergence of spatial learning. Previous research using proximal and distal cues (Rudy et al., 1987) supports this hypothesis as 
they found that rats performed well on a water maze task at p17 when proximal cues were available but were only able to perform well using distal cues at p20. In all four experiments, water maze and dry maze, only distal cues were present and similar to the research carried out by Rudy and colleagues (1987), the improvements on the task became apparent at p20. Therefore, the mechanisms needed for spatial learning using only distal cues seems to emerge later in life than the mechanisms necessary for proximal cue learning.

In accordance with the hypothesis that a cognitive-spatial process is developing in the brain, previous research (Holahan et al., 2007) examined the natural time course of mossy fiber expansion in the brain and found mossy fibers project to the SO from the SL of the CA3 between p18 and p21. Mossy fiber expansion is seen not only naturally (Holahan et al., 2007), but also following training on a water maze task (Holahan et al., 2006; Keeley et al., 2010; Wartman et al., 2012) and a dry maze task (Wartman et al., 2012). Thus, these anatomical changes that occur naturally as well as following learning on a spatial task suggest that spatial functions emerge in accordance with brain development.

\section{NMDA receptors and memory}

Long-term potentiation is the idea that high-frequency stimulation increases synaptic efficiency. The induction of LTP is mediated through NMDA receptor activation (Bliss \& Collingridge, 1993; Collingridge et al., 1983; Ge at al., 2010; Moser \& Moser, 2000). Without NMDA receptor activation, the induction of LTP is impaired (Morris et al., 1986) as well as the acquisition of spatial tasks such as the water maze (Ramirez-Amaya et al., 2001) and place learning (Morris et al., 1986). Experiment 2 
investigated the effects of a non-competitive NMDA receptor antagonist MK-801 on learning the water maze task during development. Results showed that pretreatment with MK-801 led to impairments on the water maze as rats given the antagonist showed significantly longer latencies to reach the hidden platform. This is consistent with previous research (Ahlander et al., 1999; Morris et al., 1986) showing spatial learning impairments after NMDAr blockade. However, there is research (Bannerman et al., 1995; Moser \& Moser, 2000; Saucier \& Cain, 1995) where pretraining seems to eradicate spatial learning deficits that result from NMDA receptor antagonists.

It is unclear whether NMDAr antagonists disrupt water maze performance because they are affecting the ability of the rat to learn or whether they are resulting in motor deficits. MK-801-treated rats in Groups 1 and 3 of Experiment 2 showed significantly slower speeds than saline-treated rats which is consistent with previous research (Ahlander et al., 1999). Thus, it seems that impaired swimming ability could be a contributing factor for the apparent spatial performance deficits seen in rats treated with MK-801. However, it should be noted that other research (Morris et al., 1986; Holahan et al., 2012) revealed no sensorimotor deficits when blocking NMDAr. Holahan and colleagues (2012) revealed dose-dependent differences using an appetitive operant task in rats treated with MK-801. The $0.05 \mathrm{mg} / \mathrm{kg}$ dose produced an elevated lever pressing response, where as the $0.1 \mathrm{mg} / \mathrm{kg}$ dose did not produce such a response. The group given the $0.1 \mathrm{mg} / \mathrm{kg}$ dose also showed fewer nose pokes during extinction than the $0.05 \mathrm{mg} / \mathrm{kg}$ group. The ideal dose $0.05 \mathrm{mg} / \mathrm{kg}$ was given in the current study and thus it is believed sensorimotor deficits were not the explanation for the poor spatial performance. The slower speeds could either indicate the rat was unsure of the platform location (spatial 
deficit) or the drug was affecting the rat's ability to swim to the platform successfully (motor deficit). It remains inconclusive as to whether the slower latencies indicate learning impairments or performance problems.

\section{Emergence of spatial functions}

Many studies indicate that place learning (i.e. using only distal cues to find a hidden platform) develops later in life than cue learning (Schenk, 1985; Rudy et al., 1987; Akers et al., 2007). Rats in Experiment 1 showed improvement over days with a decrease in latencies to find the hidden platform by p20. Groups in Experiment 2 that began water maze training on one of three days (p16, p18, or p20) also showed decreased latencies at p20 regardless of prior training. Therefore, prior experience with the aspects of the water maze task was not the only factor for why rats showed improvements over days on the water maze, but it seemed as though the cognitive ability for spatial learning emerged around p20.

Consistent with this, results from Experiment 3 also suggested the emergence of spatial functioning around p20 as those rats showed significantly shorter latencies than those trained at p16 and p18. As previously mentioned, it is possible the improvement was seen at p20 because the rats were physically stronger than their younger counterparts. When examining locomotor skill development, Altman and Sudarshan (1975) found that by $\mathrm{p} 15$, rats were able to perform well on a test of locomotor activity as they were capable of lifting their entire bodies and could engage in exploratory behaviours such as rearing. They also noted that rats were able to run with few slips by p16, thus the rats were more than capable to perform well on tests of locomotor activity. Therefore, performance on the dry maze was investigated. 
Our hypothesis stated that investigation ratios on the dry maze would be larger in older rats, consistent with improvements seen on the water maze in Experiments 1, 2 and 3 as well as previous research (Keeley et al., 2010; Wartman et al., 2012). The results from Experiment 4 supported this hypothesis as rats at p19 and p20 performed significantly greater than chance. The improvements around $\mathrm{p} 19 / \mathrm{p} 20$ seen on both the water maze and dry maze tasks are suggestive of cognitive development compared to previous training or physicality effects. Whether rats received previous training on the water maze or not, latencies were significantly decreased around p20. These results rule out the possibility that improvement on the water maze task was solely due to prior training or experience with the demands of the task. Rats performed well on their task around p19/p20 regardless of prior training or physical development. Therefore, motor abilities (training or physicality) do not seem to be a main contributing factor for improvement on spatial tasks during this putative sensitive developmental period. Cognitive development is likely the reason for the behavioural changes seen on both tasks around $\mathrm{p} 19 / \mathrm{p} 20$.

Immunohistochemical results from Experiments 3 and 4 showed significantly more c-Fos staining in older rats compared to their younger counterparts. Induction of cFos occurs following the activation of calcium channels (Morgan \& Curran, 1986) indicating c-Fos immunopositive cells are indicative of neuronal activation. Immediate early gene expression is important in memory and plasticity mechanisms. Therefore, the detection of c-Fos immunopositive cells in the hippocampus may be an indication of the extent to which hippocampal regions were most engaged during development and when rats performed the water maze and dry maze tasks. 
In Experiment 3, there was a significant increase in c-Fos labelling in the CA3 region of the hippocampus following learning on the water maze task compared to rats that received no training. This training effect was not seen in the CA1 region of the hippocampus. However, rats at p20 showed significantly more neuronal activation than their younger counterparts, p16 and p18 in both the CA3 and the CA1. This suggests that the hippocampal mechanisms for spatial learning may be emerging at this older age (p20). As there is little activation in the younger rats, it is possible that the mechanisms to learn spatial representations are not yet fully developed and this could explain why they performed poorly on both spatial tasks.

\section{Place cells}

The hippocampus has been noted as the brain region where spatial maps are formed. Place cells of the hippocampus are part of the neural circuits that aid in spatial navigation. This has been proposed from experiments that record and examine neuronal firing in the hippocampus when rats investigate mazes (O'Keefe \& Dostrovsky, 1971; O’Keefe \& Conway, 1978; Jung et al., 1994). O’Keefe and Dostrovsky (1971) recorded activity in the dorsal hippocampus of freely moving rats and they found that certain cells in the hippocampus fired when the rat was placed in a specific area of the maze when facing a precise direction. The finding that units in the dorsal hippocampus respond in some parts of an environment more than others has been repeatedly found (O'Keefe \& Conway, 1978; Jung et al., 1994). O’Keefe and Conway (1978) found that firing of place cells was related to the controlled cues located around the maze. Jung and colleagues (1994) noted that both the dorsal and ventral hippocampus had cells that fired when the rat moved to different locations in the search task. However, this spatial selectivity was 
stronger in CA1 neurons of the dorsal hippocampus. This led to the idea that the hippocampus and its neurons function to form a spatial map.

The dorsal hippocampus seems to respond more selectively to spatial environments and this is consistent with behavioural research that investigated the dorsal and ventral hippocampal contribution to spatial learning and memory (Moser et al., 1993). Moser and colleagues (1993) found that rats with dorsal hippocampal lesions had difficulty learning the water maze task as they showed longer latencies to find the platform during training. They also showed poor memory as they spent less time in the target quadrant during the probe test. Rats with ventral lesions did not show this impairment.

Place cells develop over pre-adolescence into adulthood (Langston et al., 2010). Researchers (Langston et al., 2010) investigated early postnatal development in an attempt to decipher when place cells begin to develop and when they morph into their adult configuration. Pups were implanted with miniature microdrives at p13 and neural activity was recorded when the rats explored an open-field maze beginning at p17. Head-direction and place cells showed adult-like properties at p17, but place cells continued to evolve from $\mathrm{p} 17$ and did not reach their full adult potential until approximately $\mathrm{p} 25-27$. The ability for rats to improve on spatial tasks such as the water maze and dry maze seen in all four experiments may be attributed to changes in their hippocampus that occur during this period of development.

The dorsal hippocampus is necessary for creating and learning a spatial map of an environment. Further research has been carried out to examine potential differential effects within hippocampal subregions (Lee et al., 2004). Lee and colleagues (2004) 
found when local and distal cues were mismatch rotated at angles greater than 45 degrees, the majority of CA3 place fields rotated on a similar track, where as the majority of CA1 place fields became altered. Therefore, neurons in the CA3 respond more accurately to altered environmental configurations than CA1 neurons. It is possible that there is differential activation of neurons in hippocampal regions in response to task demands. This differential activation was seen in Experiment 3 as rats that received water maze training showed more c-Fos immunopositive cells in the CA3 but this difference was absent in the CA1. The CA3 region seems to be more susceptible to changes in the environment (Lee et al., 2004) and this could explain why rats that received training on the water maze task showed greater activation in the CA3 than control rats. The rats undergoing water maze training were exposed to a new environment where they were learning a new task. It is possible that there was more activity in the place fields and thus, more c-Fos labelling.

\section{Mossy fibers}

Place cells are not the only component of neural circuitry that is developing at this sensitive time period. Another component that develops during this period in the hippocampus are the mossy fibers (Holahan et al., 2007). Previous research (Holahan et al., 2007) examined the natural time course of mossy fiber expansion from the SL to the SO of the CA3 region between p18 and 21. They found mossy fibers naturally expand from the SL to the SO around p18 and this persists well into adulthood in the Long-Evans rat. These structural changes in the brain occurring at this time are consistent with the improvements observed on both the water and dry maze tasks in all four experiments. 
Mossy fibers are the main input to the CA3 and they originate from the granule cells in the dentate gyrus (DG). Two primary functions of the CA3 region of the hippocampus are spatial learning and short-term memory (Kesner \& Rolls, 2001; Gilbert \& Kesner, 2003). Evidence for not only CA3 importance, but also for mossy fiber importance in spatial learning comes from research examining NMDA receptors and their role in mossy fiber projections (Holahan et al., 2007). In research carried out by Holahan and colleagues (2007), administration of NMDA receptor antagonist 3-(2carboxypiperazin-4-yl) propyl-1-phosphonic acid (CPP) led to reduced mossy fiber projections to the SO. This suggests that NMDA receptors are necessary for natural mossy fiber projections.

Mossy fiber expansion is not only seen naturally, but also following manipulations such as spatial learning. Holahan and colleagues (2006) noted that after seven days of training on the Morris water maze task, rats showed significant mossy fiber expansion to the SO. Thus, mossy fiber expansion seems to be an important morphological change in the brain when learning.

In Experiment 2, NMDA receptors were blocked in some rats and subsequently their learning was impaired. We know that NMDA receptors are necessary for proper mossy fiber expansion (Holahan et al., 2007) and that learning induces mossy fiber changes (Holahan et al., 2006), thus the inability for these morphological changes to occur in the brain may be an explanation for the poor performance seen on the water maze in rats treated with NMDA receptor antagonist MK-801. 


\section{CA1 and CA3 activation}

The hippocampal formation consists of four regions: the dentate gyrus, the hippocampus proper (CA1, CA2 \& CA3), the subicular complex, and the entorhinal cortex (Amaral \& Witter, 1989). The communication between these four regions of the hippocampal formation is largely unidirectional (Amaral \& Witter, 1989). This gives rise to the idea that regions of the hippocampal formation may perform differently in diverse tasks depending on those task demands. Immunohistochemical results from Experiments 3 and 4 showed differential activation of neurons in hippocampal regions CA1 and CA3. This suggests the potential of multiple avenues of processing information according to the task being completed.

In accordance with this view, previous research investigating odour discrimination learning found differences in c-fos mRNA expression of the CA1 and CA3 depending on the task demands (Hess et al.,1995). Rats participated in an odour discrimination task or a nose poke task. In the nose poke task, rats were trained to poke their nose (only once into each of six holes) for a reward. For the odour discrimination task, rats had to distinguish between a positive odour and a negative odour. The positive odour nose poke was reinforced (water reward) whereas a nose poke following a negative odour was punished (strobe flash). The rats that underwent odour discrimination task training had significantly increased levels of CA3 c-fos mRNA expression compared to the CA1. However, the nose poke groups showed similar expression in both regions. This suggests that these regions are differentially activated based on task demands.

There is further research regarding the differential activation of the CA3 and CA1 regions for spatial tasks. Gilbert and Kesner (2003) found that the CA3 is important in 
associative learning tasks such as the Go/No-Go task as well as an odour-place paired association task. Rats with lesions to the CA3 were severely impaired in completing these tasks. However, lesions to the CA1 did not disrupt learning on these tasks. Both of these tasks, object and odour, are associative learning tasks in which the respective stimulus is paired with a spatial location. Thus, impairments on the tasks following CA3 lesions provide evidence that this region is more involved in spatial location tasks than the CA1. In the present study, the CA1 and CA3 were both involved as the rat aged, but activation of neurons in the CA3 region was far greater than that seen in the CA1 when the rat was participating in a spatial learning task versus a control group (Experiment 3). There are a few explanations for the main effect of training observed in the CA3 region in Experiment 3. Previous research investigating the effects of lesions to the CA3 note that it is important for encoding information where learning the task takes multiple trials (Gilbert \& Kesner, 2003), for spatial learning (Gilbert \& Kesner, 2003; Lee \& Kesner, 2003), and for short-term memory (Kesner \& Rolls, 2001). Another explanation for the main effect of training in the CA3 region could be there is an increase in neuronal input from the dentate gyrus (DG) that is projecting to the CA3. Previous research has shown that spatial learning induces mossy fiber expansion from the DG to the CA3 (Holahan et al., 2007; Keeley et al., 2010 \& Wartman et al., 2012). The increased activity inferred from more c-Fos positively-labelled cells in the CA3 of the trained group may be due to an amplified input received from the DG. This hypothesis is in line with Amaral and Witter's (1989) anatomical work noting unidirectional communication from the DG to the CA3 region of the hippocampus. The one-day of training may not have been sufficiently long enough for this communication to carry on to the CA1 region of 
the hippocampus from the CA3 pyramidal cells via the Schaffer collaterals. Although the exact origin for the differential involvement of these subfields is unknown, the difference is important to note.

\section{Comparison to humans}

The water maze task requires navigational place learning to locate the hidden platform. When assessing spatial learning in children, researchers (Overman et al., 1996; Lehnung et al., 1998; Leplow et al., 2003) found that children between seven and tenyears-old could complete spatial learning tasks using distal cues. Before and at seven years of age, children were able to successfully complete the task if proximal cues were present. This work investigating spatial development in humans (Overman et al., 1996; Lehnung et al., 1998; Leplow et al., 2003) is consistent with animal research (Rudy et al., 1987). Rudy and colleagues (1987) found that rats only 17 days old were able to locate a platform when proximal cues were present, and that 20 day old rats were able to perform this task with only distal cues. This emergence was apparent in all four experiments as rats showed significant improvements on both the water maze and dry maze around p20. Spatial learning seems to emerge by p20 in rats and between seven and ten years of age in humans. Thus, it may be that the neural circuitry in the hippocampus necessary for this type of learning is not fully developed until these later ages.

\section{Limitations and future directions}

This study was carried out to identify the sensitive developmental period and discover if this time was sensitive due to physical changes/abilities or cognitive development. Two different spatial learning tasks were used to examine similarities and differences between a physically difficult and a less physically difficult task. There exists 
a limitation when comparing results from these two tasks. Although they are similar and the rat must use distal cues in both tasks to complete it successfully, they remain different tasks. The biggest distinction is that the water maze task contains a reward and the dry maze task does not. The reward in the water maze task is escaping from the water. Thus, the rats are motivated to complete the task to receive this reward, whereas the dry maze is an exploratory task with no such reward. Future research may perform a similar study examining the sensitive developmental period but instead should use a rewarding task for the less physically demanding task. A possibility for this would be the radial arm maze task which is hippocampal dependent, less physically demanding than the water maze and contains a food reward.

Another limitation in this study regards the count of c-Fos immunopositive cells. It is possible that the differences seen were due to cell density differences instead of true activation differences in the rat. We analyzed the traced area to ensure that the size of the hippocampus was not the determining factor for the increased counts seen at p20. However, we did not analyze cell density. Further research should be carried out to determine whether the CA3 and CA1 regions of the hippocampus are more densely packed at $\mathrm{p} 20$ or if the difference we see is accurate.

\section{Conclusion}

The results from this study suggest that spatial memory mechanisms rapidly emerge at p19/p20. Testing on both the water maze (with and without prior training) and the dry maze proposes that these memory mechanisms occur independently from performance factors (prior training or physicality). The increased number of c-Fos immunopositive cells in older rats (p20) confirms more hippocampal activation as the rat 
ages and thus spatial memory mechanisms are based on hippocampal function.

Therefore, the combination of both behavioural and neuroanatomical results confirm the hypothesis that $\mathrm{p} 19-\mathrm{p} 21$ represents a sensitive period for hippocampal development and modification. 


\section{References}

Ahlander, M., Misane, I., Schott, P.A., \& Ogren, S.O. 1999. A behavioral analysis of the spatial learning deficit induced by the NMDA receptor antagonist MK-801 (dizocilpine) in the rat. Neuropsychopharmacology 21, 414-426.

Akers, K.G., Candelaria, F.T., \& Hamilton, D.A. 2007. Preweanling rat solve the Morris water maze task via directional navigation. Behavioural Neuroscience 121, 14261430.

Altman, J., \& Sudarshan, K. 1975. Postnatal development of locomotion in the laboratory rat. Animal Behaviour 23, 896-920.

Amaral, D.G., \& Witter, M.P. 1989. The three-dimensional organization of the hippocampal formation: A review of anatomical data. Neuroscience 31, 571-591.

Aniksztejn, L., \& Ben-Ari, Y. 1991. Novel form of long-term potentiation produced by a $\mathrm{K}+$ channel blocker in the hippocampus. Nature 349, 67-69.

Bailey, C.H., \& Kandel, E.R. 1993. Structural changes accompanying memory storage. Annual Review of Physiology 55, 397-426.

Bannerman, D.M., Good, M.A., Butcher, S.P., Ramsay, M., \& Morris, R.G.M. 1995. Distinct components of spatial learning revealed by prior training and NMDA receptor blockade. Nature 378, 182-186.

Bayer, S.A. 1980. Development of the hippocampal region in the rat II. Morphogenesis during embryonic and early postnatal life. Journal of Comparative Neurology 190, 115-134. 
Ben-Ari, Y., \& Represa, A. 1990. Brief seizure episodes induce long-term potentiation and mossy fibre sprouting in the hippocampus. Trends in Neuroscience 13, 312317.

Bergmann, M., Post, A., Rittel, I., Bechmann, I., \& Nitsch, R. 1997. Expression of synaptophysin in sprouting neurons after entorhinal lesion in the rat. Experimental Brain Research 117, 80-86.

Blackstad, T.W. 1956. Commissural connections of the hippocampal region in the rat, with special reference to their mode of termination. Journal of Comparative Neurology 105, 417-537.

Bliss, T.V.P., \& Lomo, T. 1973. Long-lasting potentiation of synaptic transmission in the dentate area of the anaesthetized rabbit following stimulation of the perforant path. The Journal of Physiology 232, 331-356.

Bliss, T.V.P., \& Douglas, R.M., Errington, M.L., \& Lynch, M.A. 1986. Correlation between long-term potentiation and release of endogenous amino acids from dentate gyrus of anaesthetized rats. The Journal of Physiology 377, 391-408.

Bliss, T.V.P., \& Collingridge, G.L. 1993. A synaptic model of memory: Long-term potentiation in the hippocampus. Nature 361, 31-39.

Bolles, R.C., \& Woods, P.J. 1964. The ontogeny of behaviour in the albino rat. Animal Behaviour, 12, 427-441. 
Chen, P.E., \& Wyllie, D.J.A. 2006. Pharmacological insights obtained from structurefunction studies of ionotropic glutamate receptors. British Journal of Pharmacology 147, 839-853.

Clark, R.E., Zola, S.M., Squire, L.R. 2000. Impaired recognition memory in rats after damage to the hippocampus. The Journal of Neuroscience 20, 8853-8860.

Collingridge, G.L., Kehl, S.J., McLennan, H. 1983. Excitatory amino acids in synaptic transmission in the Schaffer collateral-commissural pathway of the rat hippocampus. Journal of Physiology 334, 33-46.

Collingridge, G.L., \& Bliss, T.V.P. 1987. NMDA receptors - their role in long-term potentiation. Trends in Neuroscience 7, 288-293.

Corkin, S. 1984. Lasting consequences of bilateral medial temporal lobectomy: clinical course and experimental findings in H.M. Seminars in Neurology 4, 249-259.

Cotman, C.W., Monaghan, D.T., Ganong, A.H. 1988. Excitatory amino acid neurotransmission: NMDA receptors and Hebb-type synaptic plasticity. Annual Review Neuroscience 11, 61-80.

Curran, T., Morgan, J.I. 1995. Fos: An immediate-early transcription factor in neurons. Journal of Neurobiology 26(3), 403-412.

Danscher, G. 1981. Histochemical demonstration of heavy metals. A revised version of the sulphide silver method suitable for both light and electronmicroscopy. Histochemistry 71, 1-16. 
Dix, S.L., \& Aggleton, J.P. 1999. Extending the spontaneous preference test of recognition: Evidence of object-location and object-context recognition. Behavioural Brain Research 99, 191-200.

Escobar, M.L., Barea-Rodriguez, E.J., Derrick, B.E., Reyes, J.A., Martinez Jr., J.L. 1997. Opioid receptor modulation of mossy fiber synaptogenesis: Independence from long-term potentiation. Brain Research 751, 330-335.

Fenoglio, K.A., Brunson, K.L., Baram, T.Z. 2006. Hippocampal neuroplasticity induced by early-life stress: Functional and molecular aspects. Frontiers in Neuroendocrinology 27, 180-192.

Fiala, B.A., Joyce, J.N., \& Greenough, W.T. 1978. Environmental complexity modulates growth of granule cell dendrites in developing but not adult hippocampus of rats. Experimental Neurology 59, 372-383.

Foreman, N., \& Altaha, M. 1992. The development of exploration and spontaneous alternation in hooded rat pups: Effects of unusually early eyelid opening. Developmental Psychobiology 24(7), 521-537.

Frederickson, C.J., Klitenick, M.A., Manton, W.I., \& Kirkpatrick, J.B. 1983. Cytoarchitectonic distribution of zinc in the hippocampus of man and the rat. Brain Research 273, 335-339.

Gard, C., Hard, E., Larsson, K. \& Petersson, V.A. 1967. The relationship between sensory stimulation and gross motor behaviour during the postnatal development in the rat. Animal Behaviour, 15, 563-567. 
Gaskin, S., Tremblay, A., \& Mumby, D.G. 2003. Retrograde and anterograde object recognition in rats with hippocampal lesions. Hippocampus 13, 962-969.

Gaskin, S., Tardif, M., \& Mumby, D.G. 2009. Patterns of retrograde amnesia for recent and remote incidental spatial learning in rats. Hippocampus 19, 1212-1221.

Gaskin, S., Tardif, M., Cole, E., Piterkin, P., Kayello, L., \& Mumby, D.G. 2010. Object familiarization and novel-object preference in rats. Behavioural Processes 83, 6171.

Ge, Y., Dong, Z., Bagot, R.C., Howland, J.G., Phillips, A.G., Wong, T.P., Wang, Y.T. 2010. Hippocampal long-term depression is required for the consolidation of spatial memory. Proceedings of the National Academy of Sciences 107, 1669716702.

Gilbert, P.E., \& Kesner, R.P. 2003. Localization of function within the dorsal hippocampus: The role of the CA3 subregion in paired-associate learning. Behavioural Neuroscience 117, 1385-1394.

Grover, L.M., \& Teyler, T.J. 1995. Different mechanisms may be required for maintenance of NMDA receptor-dependent and independent forms of long-term potentiation. Synapse 19, 121-133.

Hanse, E., \& Gustafsson, B. 1994. Onset and stabilization of NMDA receptor-dependent hippocampal long-term potentiation. Neuroscience Research 20, 15-25.

Herrera, D.G., \& Robertson, H.A. 1996. Activation of c-fos in the brain. Progress in Neurobiology 50, 83-107. 
Hess, U.S., Lynch, G., \& Gall, C.M. 1995. Changes in c-fos mRNA expression in rat brain during odor discrimination learning: Differential involvement of hippocampal subfields CA1 and CA3. The Journal of Neuroscience 15, 47864795.

Holahan, M.R., Rekart, J.L., Sandoval, J., \& Routtenberg, A. 2006. Spatial learning induces presynaptic structural remodelling in the hippocampal mossy fiber system of two rat strains. Hippocampus 16, 560-570.

Holahan, M.R., Honegger, K.S., \& Routtenberg, A. 2007. Expansion and retraction of hippocampal mossy fibers during postweaning development: Strain-specific effects of NMDA receptor blockade. Hippocampus 17, 58-67.

Holahan, M.R., Westby, E.P., \& Albert, K. 2012. Comparison of the MK-801-induced appetitive extinction deficit with pressing for reward and associated pERK1/2 staining in prefrontal cortex and nucleus accumbens. Behavioural Brain Research 228, 194-202.

Herdegen, T., Kovary, K., Buhl, A., Bravo, R., Zimmermann, M., \& Grass, P. 1995. Basal expression of the inducible transcription factors c-Jun, JunB, JunD, c-Fos, FosB, and Krox-24 in the adult rat brain. The Journal of Comparative Neurology 354, 39-56.

Jahn, R., Schiebler, W., Ouimet, C., \& Greengard, P. 1985. A 38,000-dalton membrane protein (p38) present in synaptic vesicles. Cell Biology 82, 4137-4141. 
Jung, M.W., Wiener, S.I., \& McNaughton, B.L. 1994. Comparison of spatial firing characteristics of units in dorsal and ventral hippocampus of the rat. Journal of Neuroscience 14, 7347-7356.

Kantor, D.B., Kolodkin, A.L. 2003. Curbing the excesses of youth: molecular insights into axonal pruning. Neuron 38, 849-852.

Kauer, J.A., Malenka, R.C., Nicoll, R.A. 1988. A persistent postsynaptic modification mediates long-term potentiation in the hippocampus. Neuron 1, 911-917.

Keeley, R.J., Wartman, B.C., Hausler, A.N., \& Holahan, M.R. 2010. Effect of juvenile pretraining on adolescent structural hippocampal attributes as a substrate for enhanced spatial performance. Learning and Memory 17, 344-354.

Kempermann, G., Kuhn, H.G., \& Gage, F.H. 1997. More hippocampal neurons in adult mice living in an enriched environment. Nature 386, 493-495.

Kesner, R.P., \& Rolls, E.T. 2001. Role of long-term synaptic modification in short-term memory. Hippocampus 11, 240-250.

Kimble, D.P. 1963. The effects of bilateral hippocampal lesions in rats. Journal of Comparative and Physiological Psychology 56, 273-283.

Langston, R.F., Ainge, J.A., Couey, J.J., Canto, C.B., Bjerknes, T.L., Witter, M.P., Moser, E.I., \& Moser, M-B. 2010. Development of the spatial representation system in the rat. Science $328,1576-1580$. 
Lee, I., Yoganarasimha, D., Rao, G., \& Knierim, J.J. 2004. Comparison of population coherence of place cells in hippocampal subfields CA1 and CA3. Nature 430, 456-459.

Lehnung, M., Leplow, B., Friege, L., Herzog, A., Mehdorn, M., \& Ferstl, R. 1998. Development of spatial memory and spatial orientation in preschoolers and primary school children. British Journal of Psychology 89, 463-480.

Leplow, B., Lehnung, M., Pohl, J., Herzog, A., Ferstl, R., \& Mehdorn, M. 2003. Navigational place learning in children and young adults as assessed with a standardized locomotor search task. British Journal of Psychology 94, 299-317.

Li, S., Reinprecht, I., Fahnestock, M., \& Racine, R.J. 2002. Activity-dependent changes in synaptophysin immunoreactivity in hippocampus, piriform cortex, and entorhinal cortex of the rat. Neuroscience 115, 1221-1229.

Lynch, G.S., Gribkoff, V.K., \& Deadwyler, S.A. 1976. Long term potentiation is accompanied by a reduction in dendritic responsiveness to glutamic acid. Nature $263,151-153$

Malenka, R.C. 1991. Postsynaptic factors control the duration of synaptic enhancement in area CA1 of the hippocampus. Neuron 6, 53-60.

Malenka, R.C. 1994. Synaptic plasticity in the hippocampus: LTP and LTD. Cell 78, $535-538$. 
Maletic-Savatic, M., Malinow, R., \& Svoboda, K. 1999. Rapid dendritic morphogenesis in CA1 hippocampal dendrites induced by synaptic activity. Science, 283(5409), 1923-1927.

Markus, E.J., Petit, T.L., \& LeBoutillier, J.C. 1987. Synaptic structural changes during development and aging. Developmental Brain Research 35, 239-248.

Masliah, E., Terry, R.D., Alford, M., \& DeTeresa, R. 1990. Quantitative immunohistochemistry of synaptophysin in human neocortex: An alternative method to estimate density of presynaptic terminals in paraffin sections. The Journal of Histochemistry and Cytochemistry 38, 837-844.

Masliah, E., Mallory, M., Hansen, L., DeTeresa, R., \& Terry, R.D. 1993. Quantitative synaptic alterations in the human neocortex during normal aging. Neurology 43, 192-197.

Milner, B., Squire, L.R. \& Kandel, E.R. 1998. Cognitive neuroscience and the study of memory. Neuron 20, 445-468.

Morgan, J.I., \& Curran, T. 1986. Role of ion flux in the control of c-Fos expression. Nature 322, 552-555.

Morris, R.G.M., Garrud, P., Rawlins, J.N.P., \& O’Keefe, J. 1982. Place navigation impaired in rats with hippocampal lesions. Nature 297, 681-683.

Morris, R.G.M., Anderson, E., Lynch, G.S., \& Baudry, M. 1986. Selective impairment of learning and blockade of long-term potentiation by an N-methyl-D-aspartate receptor antagonist, AP5. Nature 319, 774-776. 
Morris, R.G.M. 1989. Synaptic plasticity and learning: Selective impairment of learning in rats and blockade of long-term potentiation in vivo by the N-methyl-Daspartate receptor antagonist AP5. The Journal of Neuroscience 9, 3040-3057.

Moser, E., Moser, M-B., \& Andersen, P. 1993. Spatial learning impairment parallels the magnitude of dorsal hippocampal lesions, but is hardly present following ventral lesions. The Journal of Neuroscience 13, 3916-3925.

Moser, M-B., \& Moser, E.I. 2000. Pretraining and the function of hippocampal long-term potentiation. Neuron 26, 559-561.

Mumby, D.G., Gaskin, S., Glenn, M.J., Schramek, T.E., \& Lehmann, H. 2002. Hippocampal damage and exploratory preferences in rats: Memory for objects, places, and contexts. Learning and Memory 9, 49-57.

Nadel, L., \& Bohbot, V. 2001. Consolidation of memory. Hippocampus 11, 56-60.

Nakanishi, S., \& Masu, M. 1994. Molecular diversity and functions of glutamate receptors. Annual Review of Biophysics and Biomolecular Structure 23, 319-348.

O’Keefe, J., \& Dostrovsky, J. 1971. The hippocampus as a spatial map. Preliminary evidence from unit activity in the freely-moving rat. Brain Research 34, 171-175.

O’Keefe, J., \& Conway, D.H. 1978. Hippocampal place units in the freely moving rat: Why they fire where they fire. Experimental Brain Research 31, 573-590.

Overman, W.H., Pate, B.J., Moore, K., \& Peuster, A. 1996. Ontogeny of place learning in children as measure in the radial arm maze, Morris search task, and open field task. Behavioural Neuroscience 110, 1205-1228. 
Petit, T.L., LeBoutillier, J.C., Gregorio, A., \& Libstug, H. 1988. The pattern of dendritic development in the cerebral cortex of the rat. Developmental Brain Research 41, 209-219.

Raisman, G., Cowan, W.M. \& Powell, T.P.S. 1965. The extrinsic afferent, commissural and association fibres of the hippocampus. Brain 88, 963-996

Ramirez-Amaya, V., Escobar, M.L., Chao, V., \& Bermudez-Rattoni, F. 1999.

Synaptogenesis of mossy fibers induced by spatial water maze overtraining. Hippocampus 9, 631-636.

Ramirez-Amaya, V., Balderas, I., Sandoval, J., Escobar, M.L., \& Bermudez-Rattoni, F. 2001. Spatial long-term memory is related to mossy fiber synaptogenesis. The Journal of Neuroscience 21, 7340-7348.

Rudy, J.W., Stadler-Morris, S., \& Albert, P. 1987. Ontogeny of spatial navigation behaviours in the rat: Dissociation of "proximal"-and "distal"-cue-based behaviours. Behavioral Neuroscience 101, 62-73.

Salt, T.E., \& Eaton, S.A. 1996. Functions of ionotropic and metabotropic glutamate receptors in sensory transmission in the mammalian thalamus. Progress in Neurobiology 48, 55-72.

Saucier, D., \& Cain, D.P. 1995. Spatial learning without NMDA receptor-dependent long-term potentiation. Nature 378, 186-189.

Schenk, F. 1985. Development of place navigation in rats from weaning to puberty. Behavioural and Neural Biology 43, 69-85. 
Scoville, W.B., \& Milner, B. 1957. Loss of recent memory after bilateral hippocampal lesions. The Journal of Neuropsychiatry and Clinical Neurosciences 12, 11-21.

Storm, J.F. An after-hyperpolarization of medium duration in rat hippocampal pyramidal cells. Journal of Physiology 409, 171-190.

Sur, M., Rubenstein, J.L. 2005. Patterning and plasticity of the cerebral cortex. Science $310,805-810$.

Teyler, T.J. \& DiScenna, P. 1984. The topological anatomy of the hippocampus: A clue to its function. Brain Research Bulletin 12, 711-719.

Thomas, L., \& Betz, H. 1990. Synaptophysin binds to physophilin, a putative synaptic plasma membrane protein. The Journal of Cell Biology 111, 2041-2052.

Thompson, S.H. 1997. Three pharmacologically distinct potassium channels in molluscan neurones. Journal of Physiology 265, 465-488.

Tischmeyer, W., Kaczmarek, L., Strauss, M., Jork, R., \& Matthies, H. 1990.

Accumulation of c-fos mRNA in rat hippocampus during acquisition of a brightness discrimination. Behavioural and Neural Biology 54, 165-171.

Uekita, T., \& Okaichi, H. 2009. Pretraining does not ameliorate spatial learning deficits induced by intrahippocampal infusion of AP5. Behavioural Neuroscience 123, $520-526$.

Wartman, B.C. Gervais, N.J., Smith, C., Comba, R., Mumby, D.G., \& Holahan, M.R. 2012. Enhanced adolescent learning and hippocampal axonal projections 
following preadolescent spatial exposure to a water or dry maze. Brain Research $1475,37-48$.

Wisden, W., \& Seeburg, P.H. 1993. Mammalian ionotropic glutamate receptors. Current Opinion in Neurobiology 3, 291-298.

Wozniak, D.F., Olney, J.W., Kettinger, L. $3^{\text {rd }}$., Price, M., Miller, J.P. 1990. Behavioural effects of MK-801 in the rat. Psychopharmacology 101, 47-56. 